Review

\title{
Ciguatera in the Indian Ocean with Special Insights on the Arabian Sea and Adjacent Gulf and Seas: A Review
}

\author{
Nazima Habibi ${ }^{1}\left(\mathbb{D}\right.$, Saif Uddin ${ }^{1, *(\mathbb{D}}$, Marie-Yasmine Dechraoui Bottein ${ }^{2} \mathbb{C}$ and Mohd Faizuddin ${ }^{3}$ \\ 1 Environment and Life Sciences Research Center, Kuwait Institute for Scientific Research, Safat 13109, Kuwait; \\ nhabibi@kisr.edu.kw \\ 2 Université Côte d'Azur, CNRS, ECOSEAS, UMR7035, Parc Valrose, 06108 Nice, France; y.bottein@gmail.com \\ 3 Gulf Geoinformation Solutions, Sharjah, United Arab Emirates; faiz_ud_din@yahoo.com \\ * Correspondence: sdin@kisr.edu.kw; Tel.: +965-24989224
}

Citation: Habibi, N.; Uddin, S.; Bottein, M.-Y.D.; Faizuddin, M. Ciguatera in the Indian Ocean with Special Insights on the Arabian Sea and Adjacent Gulf and Seas:

A Review. Toxins 2021, 13, 525. https://doi.org/10.3390/ toxins 13080525

Received: 2 July 2021

Accepted: 22 July 2021

Published: 27 July 2021

Publisher's Note: MDPI stays neutral with regard to jurisdictional claims in published maps and institutional affiliations.

Copyright: (c) 2021 by the authors. Licensee MDPI, Basel, Switzerland. This article is an open access article distributed under the terms and conditions of the Creative Commons Attribution (CC BY) license (https:// creativecommons.org/licenses/by/ $4.0 /)$.

\begin{abstract}
The dinoflagellates of the genus Gambierdiscus are found in almost all oceans and seas between the coordinates $35^{\circ} \mathrm{N}$ and $35^{\circ} \mathrm{S}$. Gambierdiscus and Fukuyoa are producers of ciguatoxins (CTXs), which are known to cause foodborne disease associated with contaminated seafood. The occurrence and effects of CTXs are well described in the Pacific and the Caribbean. However, historically, their properties and presence have been poorly documented in the Indian Ocean (including the Bay of Bengal, Andaman Sea, and the Gulf). A higher occurrence of these microorganisms will proportionately increase the likelihood of CTXs entering the food chain, posing a severe threat to human seafood consumers. Therefore, comprehensive research strategies are critically important for developing effective monitoring and risk assessments of this emerging threat in the Indian Ocean. This review presents the available literature on ciguatera occurrence in the region and its adjacent marginal waters: aiming to identify the data gaps and vectors.
\end{abstract}

Keywords: the Indian Ocean; Arabian sea; Kuwait bay; Aden Gulf; Red Sea; Gulf of Aqaba; Andaman Sea; Bay of Bengal; seafood safety; foodborne disease

Key Contribution: This review highlights the paucity of data on ciguatoxins from the vast Indian Ocean region, which is home to an enormous mass of seafood-consuming populations. Furthermore, it exports fisheries to Europe and North America, which resulted in toxic fish being found during 2012, 2015, and 2016. The monitoring of Gambierdiscus and Fukuyoa is not taken up regularly despite their recorded presence all over the Indian Ocean.

\section{Introduction}

Ciguatera poisoning $(\mathrm{CP})$ is a syndrome caused by ingestion of coral reef fish and shellfish of tropical and subtropical regions, which has caused global concern. Some dinoflagellate species of the genera Gambierdiscus and Fukuyoa are known to produce ciguatoxins (CTXs); the organisms that consume these toxic algae accumulate CTXs that are transferred and biotransformed along the marine and human food chain. These lipidsoluble and heat-resistant toxins cause gastrointestinal, cardiovascular, and neurological disorders among humans consuming the CTX-contaminated seafood [1-5]. According to a study published in 2008, CP disease estimations are uncertain and often misdiagnosed [6]. However, few reports predict 2-10 million people are annually affected by CP $[7,8]$.

Ciguatera poisoning is regarded globally as the most significant non-bacterial poisoning associated with fish consumption. It is usually limited to the consumption of toxic fish from regions between the latitudes $35^{\circ} \mathrm{N}$ and $35^{\circ} \mathrm{S}$ [9-17]. Studies have shown a strong positive correlation between Gambierdiscus abundance and algal macrophytes [18]. Some earlier studies proposed a standardized methodology for estimating Gambierdiscus abundance based on sampling macroalgae [19-21]. More recent studies brought to light the significant biases in Gambierdiscus cell distribution within the macrophytes. A 33-150\% 
variation was reported among replicates [22-24]. Despite these limitations, some first-order estimates on Gambierdiscus distributions for large geographic regions have been attempted using the average abundances $[11,25]$. These have shown a global distribution spreading across oceans with $85 \%$ of Gambierdiscus density estimates $<1000$ cells $\mathrm{g}^{-1}$ wet weight algae, with $<10 \%$ occurrence with $10-10,000$ cells $\mathrm{g}^{-1}$ wet weight algae and $<5 \%$ incidences

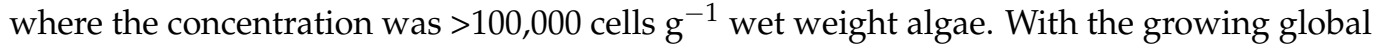
demands for seafood and international trade ease, $\mathrm{CP}$ concerns the population beyond the endemic regions and is increasingly becoming a global issue. Moreover, a greater threat is posed as there are no current antidotes for CP [26].

Historically, poisoning associated with seafood consumption was reported in different parts of the globe. It was first recounted in the West Indies as early as 1511 [27] and in the Gulf of Guinea in 1521, killing the Captains of the Spanish army [28]. It was also reported in the islands of the Indian Ocean in 1601 and various archipelagos of the Pacific Ocean in 1606 [27]. Additionally, in 1786, the surgeon of HMS Endeavour, en route to Australia, New Zealand, and the Pacific Islands, reported that the ship's Captain was poisoned by ciguatoxins [29]. A year later, the ingestion of a local gastropod (Livonia sp.) was discovered to induce neurological symptoms in the Antilles of the Caribbean Sea [30]. In 1948, the organism Gambierdiscus (originally referred to as Goniodoma sp.) was described for the first time in Cabo Verde [31]. The term "ciguatera poisoning" was coined in Cuba (Caribbean Sea) after the ingestion of a marine snail (Turbo pica) locally known as cigua [32]. The historical facts suggested the tropical and subtropical Pacific and Indian Ocean insular regions, the tropical Caribbean region, and the continental reefs to be endemic to ciguatera [27].

In recent years, there has been an increase in the frequency of reports of toxic and harmful benthic algal blooms, predominantly Gambierdiscus with the presence of Fukuyoa and Ostreopsis blooms, throughout the world [33,34]. The interest in Gambierdiscus blooms has been heightened because of increased awareness of the effects of $\mathrm{CP}$ on human health. A growth in species abundance has already been observed in subtropical and temperate regions with the threat of global warming expected to further exacerbate the situation [35-38]. However, a recent article by Hallegraeff et al. [39] suggested that intensified monitoring efforts and heightened aquaculture activities are responsible for these perceived increases in HABs events that are not underpinned to be expanded as an empirical assumption. Ciguatoxins are reported globally, being described from new sites in the Canary Islands, Indian Ocean, Japan, and Western Gulf of Mexico [8,40-45]. Until recently, the records of Gambierdiscus in the Indian Ocean were scarce and restricted to the western tropical region, whereas now its presence has been found in the Northern part of the ocean [42]. There is paucity of information on ciguatera phenomena, including the occurrence of human poisonings, of toxins in seafood, and of the causative organisms, in the Indian Ocean in general, and the northern part in particular. In the present review, we gathered the published information on reported occurrences of Gambierdiscus and identified the research gaps related to its monitoring as a tool to manage this emerging hazard.

\section{Results}

\subsection{Environmental and Global Pressures in the Northern Part of the Indian Ocean}

The Indian Ocean is the third largest ocean enclosing densely populated landmasses: In the North, India, Bangladesh, Burma, Thailand, Pakistan, Iran, and Oman among others. It also includes regional seas in the North such as the Arabian Sea and the adjacent Lakshadweep Sea, Aden Gulf in the Red Sea, the Gulf of Aqaba and Suez, the Bay of Bengal and the Andaman Sea, as well as the Gulf of Oman and the Persian Gulf further north. Countries from the Indian Ocean region have experienced unusual climatological conditions such as cyclones, El Niño-Southern Oscillation events, and coral reef bleaching during the past two decades [46-48]. Benthic microalgae are particularly influenced by these disturbances especially the coral mortality, which provides a good substrate for the formation of algal turfs and associated epiphytes [49]. The study conducted by 
Quod et al. [46] reported a significant increase in the CP causative organisms in comparison to those reported in 1980 [47], following the coral bleaching event in 1998, thus posing an incremental risk of HABs in the region and drawing detrimental consequences to the marine biodiversity and human health [50].

The unfavorable effects of increasing atmospheric levels of carbon dioxide $\left(\mathrm{CO}_{2}\right)$ and other greenhouse gases in the Indian Ocean and its marginal seas are leading towards acidification of the marine environment [48,51-53]. Supporting evidence for increased $\mathrm{CO}_{2}$ sequestration was drawn from increased marine primary productivity over the past decade $[51,54]$. The eutrophication of Kuwait bay and the nearby water bodies due to upwelling events is considered to be a predominant factor influencing the onset of algal blooms due to enriched nutrient conditions [54]. Other potential factors that have influenced the onset of blooms consist of coral bleaching, unusual variances in temperature, and calm conditions. Additional factors such as dust storms that carry micronutrients, domestic and industrial inputs, natural meteorological and oceanographic forcings, and the introduction of invasive species from ballast water discharge may all play a major role in the onset and expansion of HABs [41,45,55-58].

\subsection{Ciguatera Causative Organisms Occurrence}

The dinoflagellates Gambierdiscus and Fukuyoa are the causative organisms of CP worldwide [5]. The genus Gambierdiscus has a pantropic distribution with about 18 known species while Fukuyoa has 3 known species. Within the Indian Ocean, Gambierdiscus are more dominant in the western part. Of the several known Gambierdiscus sp., initially only Gambierdiscus toxicus was reported in Mayotte since G. toxicus was the first species that was described [50,59-61]. Later it was reported in La Reunion and Mauritius [62-65] as well (Figure 1). Gambierdiscus toxicus was also found in Mbudya Island, Oysterbay, Bawe Island, and Makoba Islands in Tanzania at depths of 5-10 m and temperatures ranging from 25 to $32{ }^{\circ} \mathrm{C}$ [66] (Figure 2). Thereafter, Gambierdiscus yasumotoi (now Fukuyoa yasumotoi (M.J. Holmes) [67]) and Gambierdiscus belizeanus were confirmed to occur in Mayotte in the Comoros archipelago [50] (Figure 3).

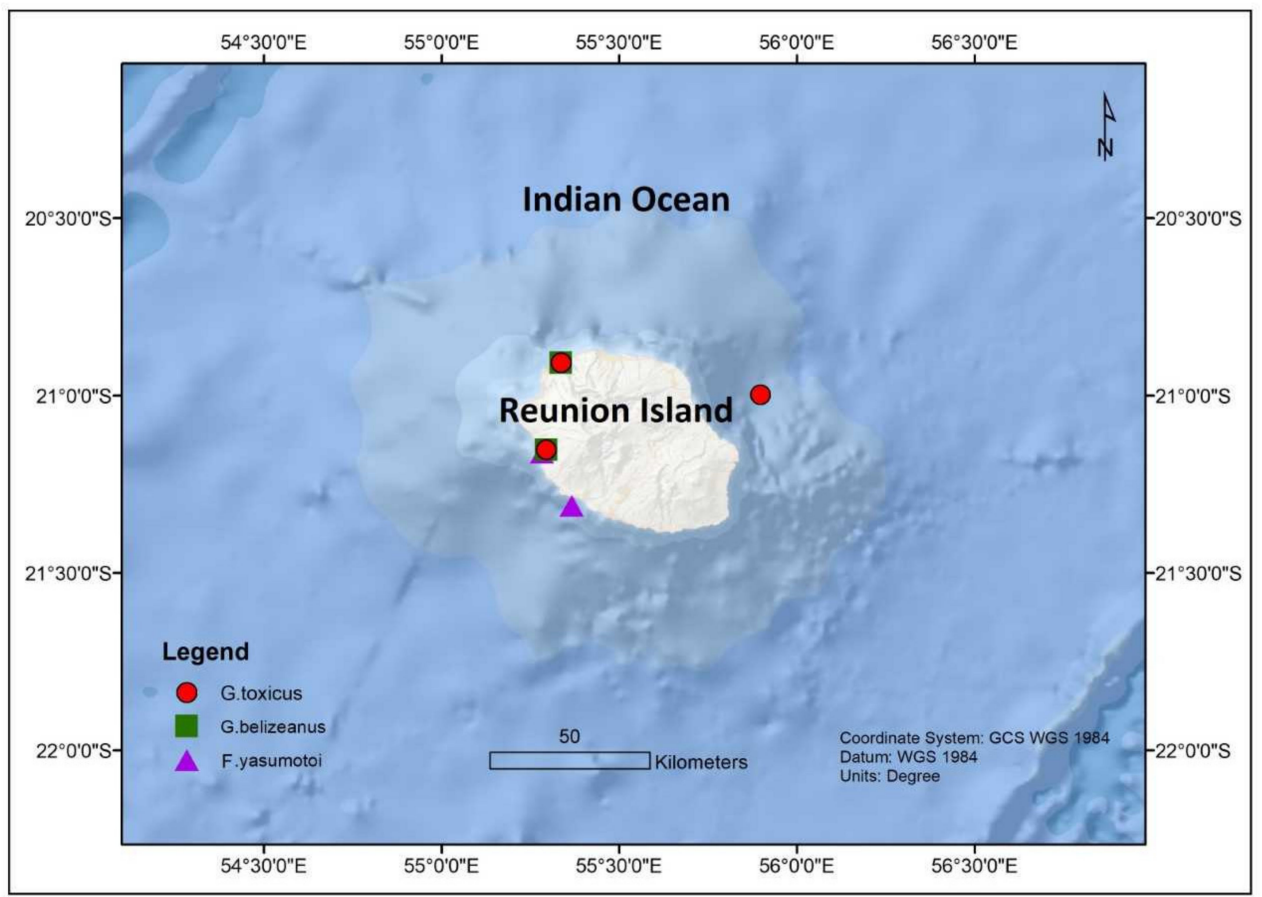

Figure 1. Spatial distribution of Gambierdiscus and Fukuyoa sp. from Reunion Island. 


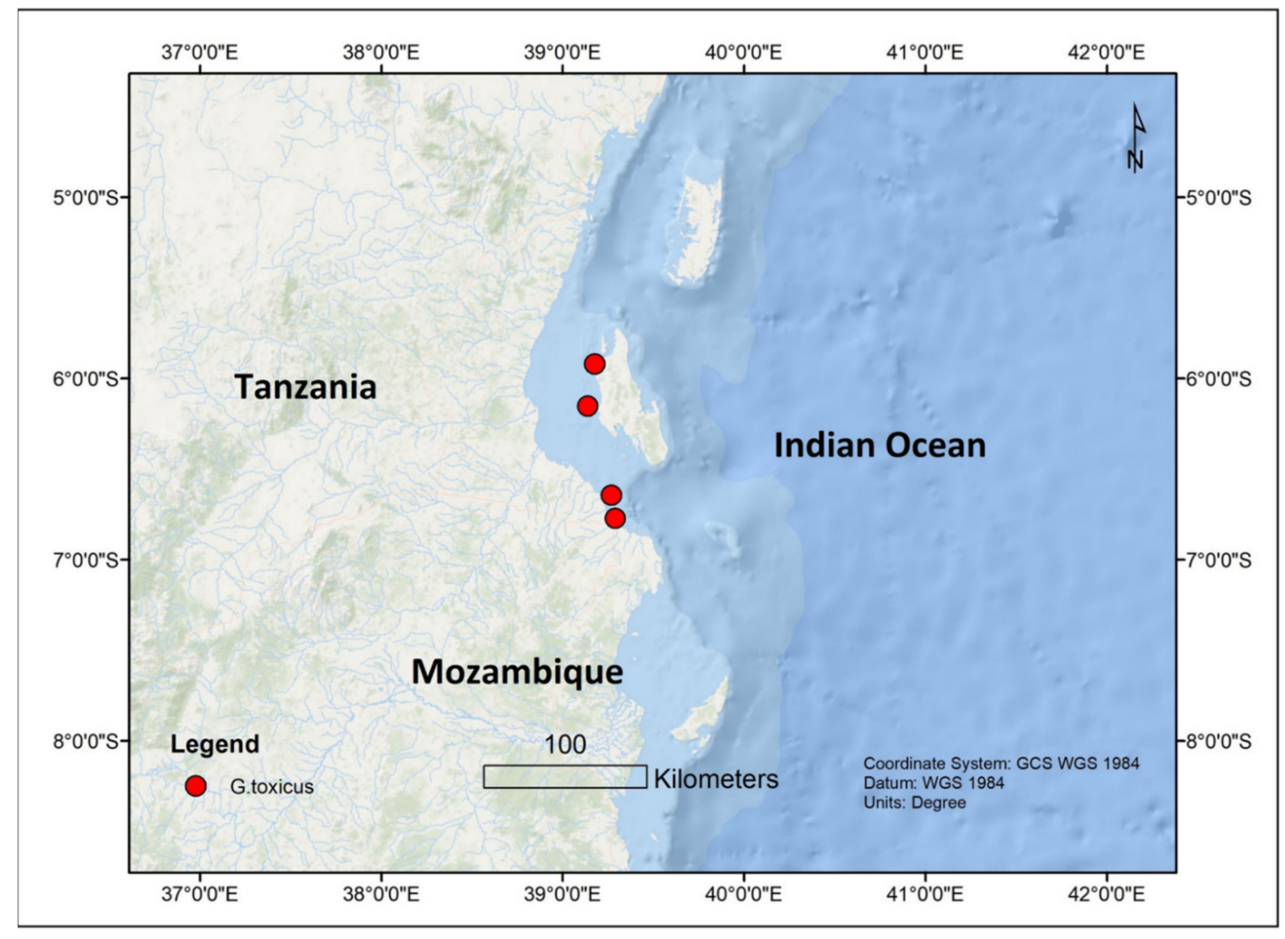

Figure 2. The occurrence of Gambierdiscus sp. from Tanzanian coastal area.

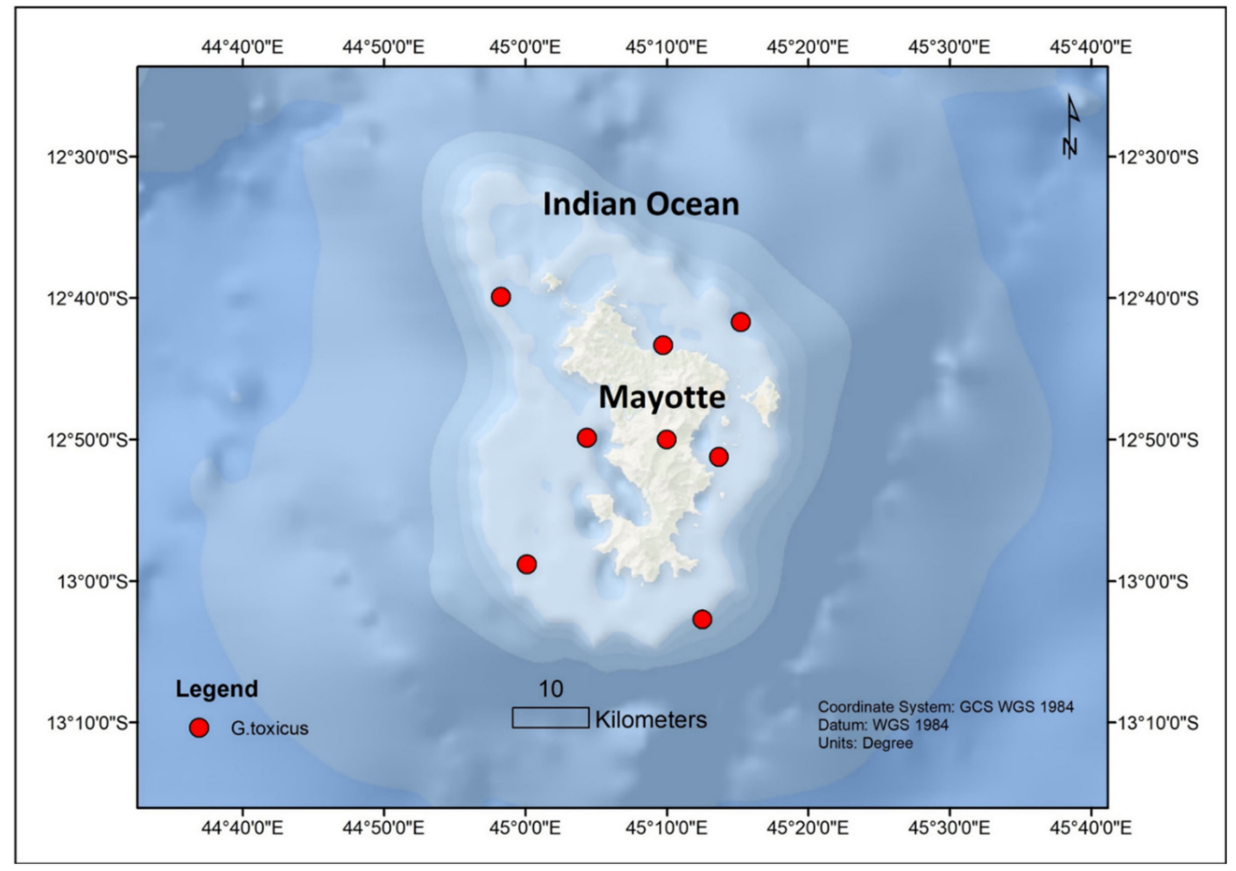

Figure 3. Gambierdiscus sp. reported from Mayotte Island, Indian Ocean.

In the northern part of the Indian Ocean, Gambierdiscus species have been noticed in the marginal seas of the Indian Ocean, such as the Arabian Sea [41,42,45,68]; Red Sea [41-45]; Gulf of Aqaba [42]; and Manora Channel, Pakistan [69]. An assessment carried out in Kuwait (Persian Gulf) from November 2012 to March 2013 showed the presence of G. yasumotoi in the shallow lagoons of Qit'at Julai'ah and southern coastal waters of Kuwait at 1-3 m depth $[68,70]$ (Figure 4). The average seawater temperatures and salinity during this time were between $23.5-25^{\circ} \mathrm{C}$ and $41.2-42.4 \mathrm{ppt}$, respectively. In another study, G. toxicus was also identified in Kuwait's territorial water [41,45] (Figure 4). 


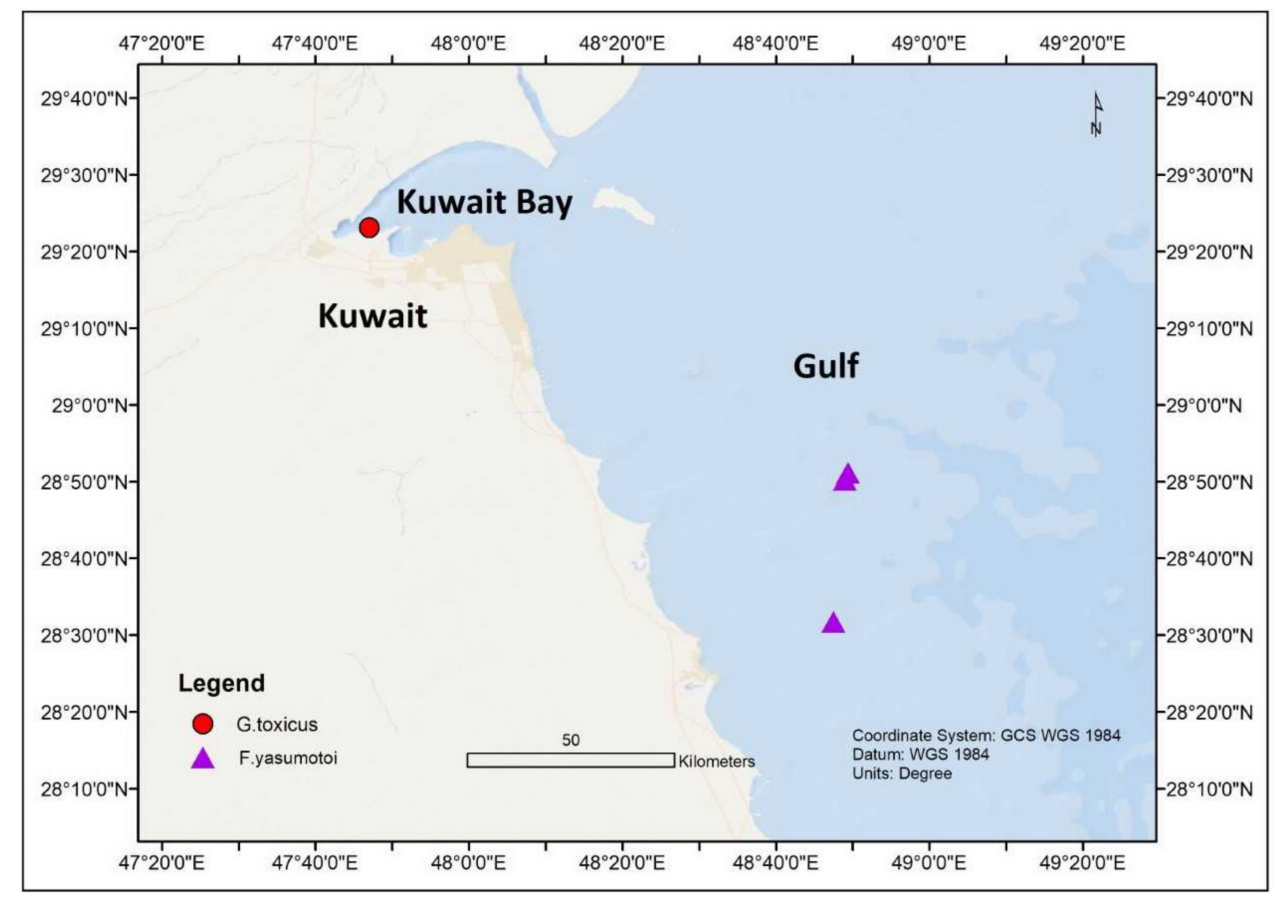

Figure 4. Gambierdiscus toxicus and Fukuyoa yasumotoi occurances in Kuwait Coastal waters.

In an investigation from the Gulf of Aqaba, Jordan during October 2010, with a seawater temperature range of $24-25^{\circ} \mathrm{C}$ and salinity of $40 \mathrm{ppt}, \mathrm{G}$. belizeanus was reported from a depth of 1.5-2.0 m (Figure 5). The toxigenic G. belizeanus was reported from the Red Sea, Saudi Arabian coast during February 2012 and May 2013 [43,44] at a sampling depth between $0.4-0.8 \mathrm{~m}$, with seawater temperatures and salinity range of $28.9-38.4{ }^{\circ} \mathrm{C}$ and 36.1-38.4 ppt respectively. The oxygen saturation during this period was very variable between $1.73-32.1 \%$.

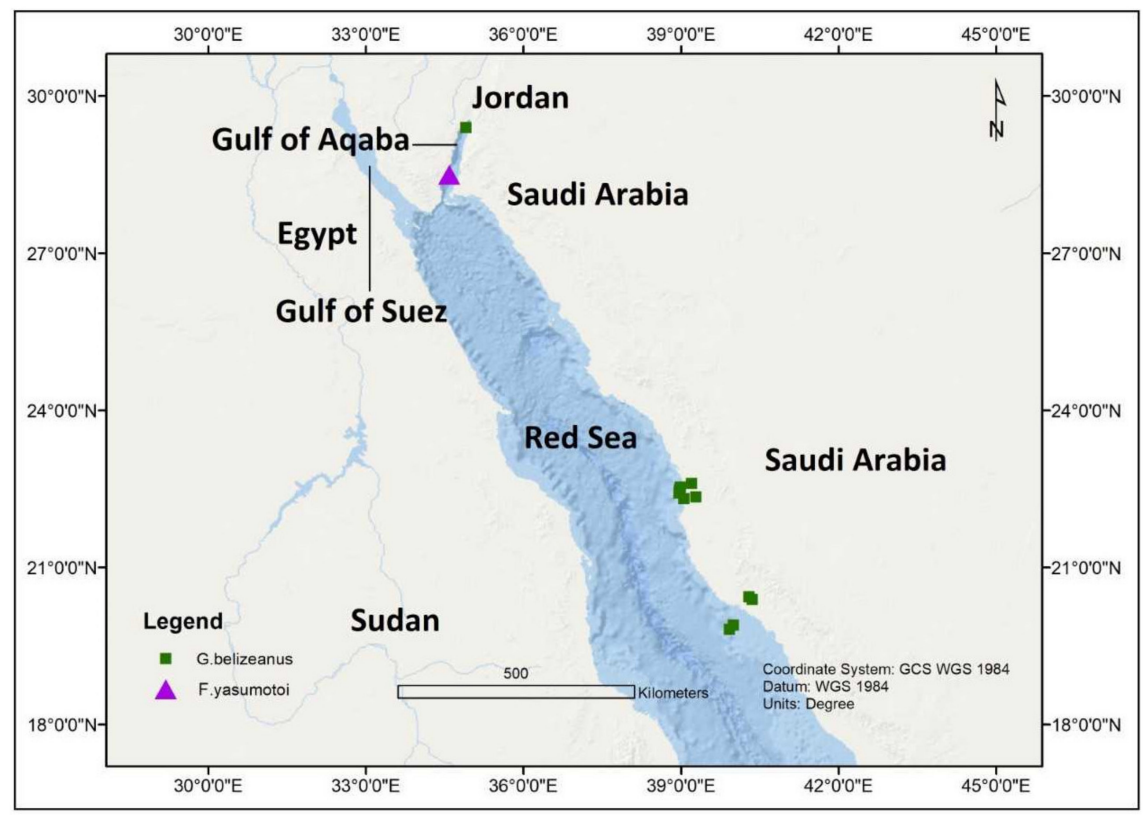

Figure 5. The Spatial distribution of Gambierdiscus belizeanus and Fukuyoa yasumotoi occurances in Red Sea and adjacent areas.

In 2011, four Gambierdiscus species including G. toxicus, G. belizeanus, G. polynesiensis, G. australes, and Fukuyoa yasumotoi were also reported from the coastal waters of Pakistan in the northern Indian Ocean [69] (Figure 6). An unidentified Gambierdiscus sp. 
was also discovered in middle of the Bay of Bengal [71] (Figure 7). In Mangalore at the southwest coast of India, CP was first described during June 2015 outbreak involving red snapper(Lutjanus bohar) [72,73], and a second outbreak during September 2016 involving red snapper again [74]. In another incidence, $\mathrm{CP}$ was also reported from Trivandrum, India [75].

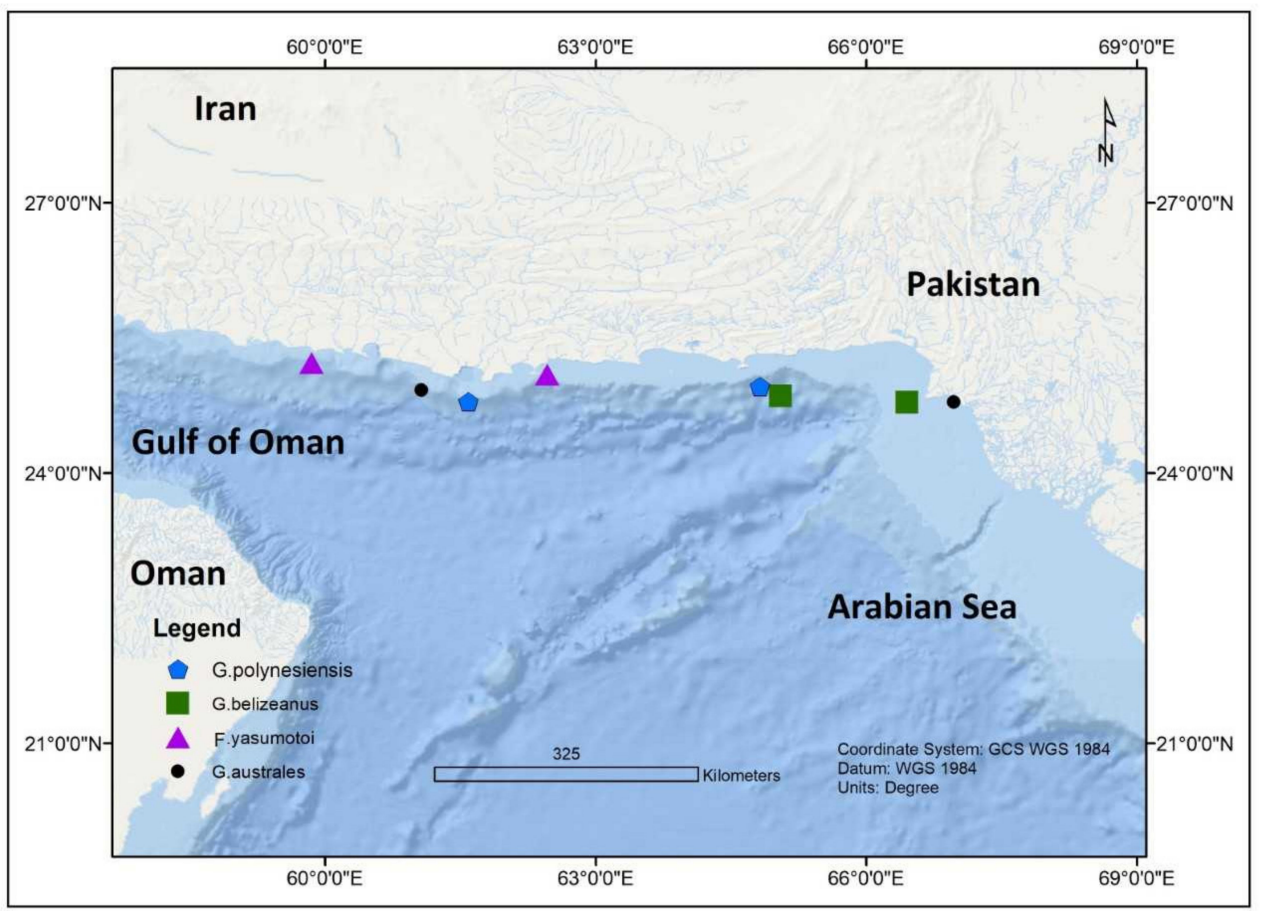

Figure 6. Occurrences of Gambierdiscus in Gulf of Oman and Pakistan's coastal regions.

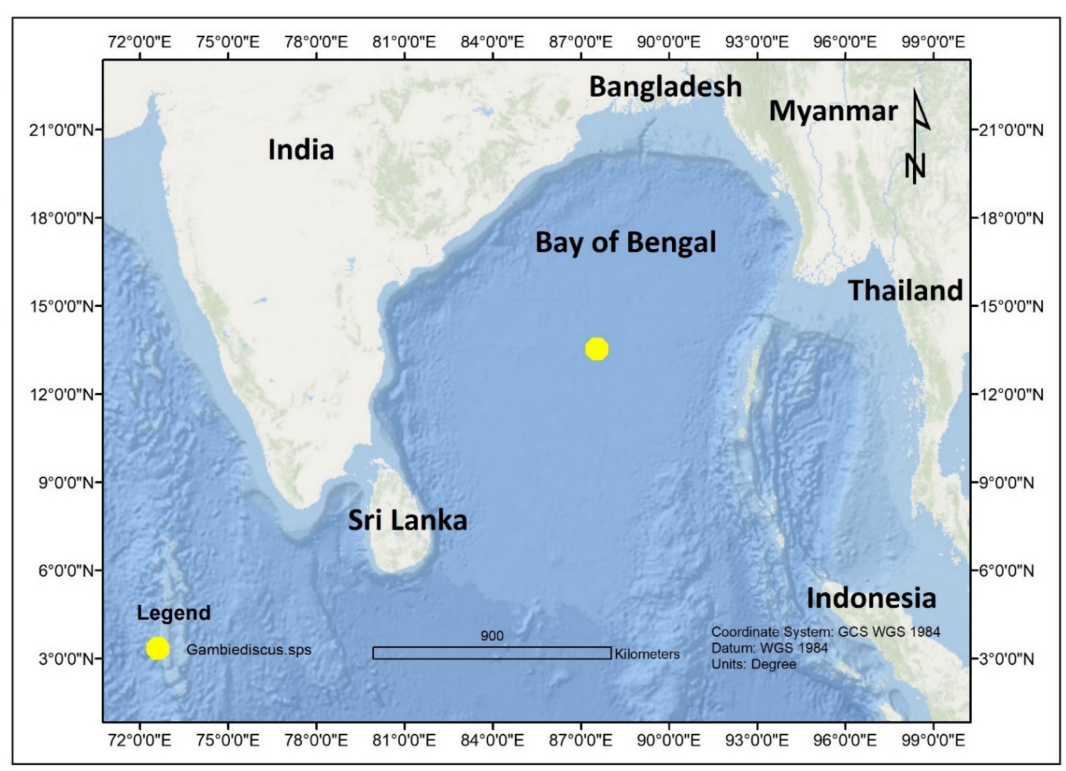

Figure 7. Gambierdiscus sp. reported from the Bay of Bengal, Indian Ocean.

\section{Discussion}

3.1. Toxin Production by Ciguatera in Indian Ocean Region and Adjoining Marginal Seas

Ciguatoxins form a large group of toxins of 40 confirmed or suspected chemical analogs. They are classified into four types, according to both their geographical origin 
and chemical structure: The Pacific CTXs (P-CTX) of the type CTX3C and CTX4A, the Caribbean CTXs (C-CTX type), and the Indian CTXs (I-CTX type) [8,27]. The existence of the I-CTX type is still speculative and may be similar to that of the C-CTX. Their structural characteristic has not yet been elucidated. Six derivatives I-CTX 1-6 were identified with a molecular weight in Dalton of 1140.6, 1156.6, 1138.6, and 1154.6, however, their chemical structure is still unknown. Four of them were retrieved from a highly toxic Lutjanus sebae (Red Emperor) from the coast of the Republic of Mauritius through optimized gradient reversed-phase high-performance liquid chromatography-electrospray ionization mass spectrometry (LC/MS) methods, in combination with a radioligand receptor binding assay (r-RBA) [76,77], and two from in a bull shark (Carcharhinus leucas) implied in a fatal intoxication in Madagascar [76-78]. The lipid-soluble extracts retrieved from the edible fishes were reported to possess CTX activity and induced lethal symptoms in mice [77] and responded to the cell bioassay [78].

The toxicity of these Gambierdiscus species collected in the Pacific and/or the Caribbean was found to vary by over 2 orders of magnitude $[11,79,80]$. It remains to be confirmed that the same ranges are observed in the Indian ocean where only a few species have been analyzed for their toxin contents. Using in vitro cytotoxicity cell-based assays (CBA with neuroblastoma with N2a cells), the toxicity of two isolates of G. belizeanus from the Red Sea was estimated at $6.50 \times 10^{-5} \mathrm{pg}$ P-CTX-1 eq. cell ${ }^{-1}$ for RS2-B6 and $1.02 \times 10^{-5} \mathrm{pg}$ P-CTX-1 eq. cell ${ }^{-1}$ for RS3-B8. Toxin production was slightly higher in G. belizeanus strains from Tahiti $\left(0.0246\right.$ fg P-CTX-3C eq. cell $\left.{ }^{-1}\right)[4,43,44]$. As compared to G. belizeanus, the species of G. polynesiensis-0.017-4.4 pg P-CTX-3C eq. cell ${ }^{-1}$; G. toxicus-0.028 pg P-CTX-3C eq. cell $^{-1}$ [4]; and G. australes- 0.04 pg CTX-1 eq cell ${ }^{-1}$ [40] are more toxigenic.

More recently, Gambieric acid D was identified for the first time in the flesh of a bull shark (Carcharhinus leucas) employing the technique of liquid chromatography coupled with high-resolution mass spectrometry (HRMS). The toxicity was confirmed through mouse bioassays (Lowest dose $=72 \mathrm{mg}$ equiv. stomach per mouse of 20-22 g) and neuro-2a cellbased assays (flesh-0.06 $\mu \mathrm{g}$ P-CTX-1 equiv./kg; stomach-92.78 $\mu \mathrm{g}$ P-CTX-1 equiv. $/ \mathrm{kg}$; fins $1-0.12 \mu \mathrm{g}$ P-CTX-1 equiv. $/ \mathrm{kg}$; and fin 3- $\mathrm{\mu g}$ P-CTX-1 equiv. $/ \mathrm{kg}$ ) [78]. Ciguatoxins were identified as CTX1B and 2,3-dihydroxy CTX3C; 51-hydroxy CTX3C; or positive by cell assay in Snapper fish (Lutjanidae) caught in the Indian Ocean and involved in 5 CP outbreak [81]. Fish samples from Sri Lanka were analyzed by the European Union Reference Laboratory for Marine Biotoxins (EURLMB) in Vigo, Spain, and the putatively contaminated fish samples were analyzed by liquid chromatography-tandem mass spectrometry (LC-MS/MS) according to a method published by [82] with slight modifications. The only available analytical standard was P-CTX-1B. Seven out of 11 samples tested positive for P-CTX-1B. In addition, other putative CTX variants were detected across most samples, however, they could not be confirmed as CTXs due to the lack of reference compounds. There are incidences of ciguatera poisoning due to the import of contaminated fish from the Indian ocean region, mainly India and Sri Lanka.

It is important to note that the investigations conducted so far have been limited in spatial coverage. Additional research is, therefore, required at a broader spatial scale to make meaningful conclusions regarding the occurrence, abundance, and temporal variability of ciguatoxins in the region.

\subsection{Ciguatera Causative Organism's Abundance}

Globally, ciguatera causative organisms' densities have been found to vary from few cells to thousands of cells per gr of substrate. Gambierdiscus is known as a slow-growing species compared to many other dinoflagellates; it takes about 5 months to increase its abundance. Chinain et al., [4] found a mean growth rate in G. polynesiensis as $0.13 \pm 0.03 \mathrm{div} \mathrm{d}^{-1}$ at exponential phase in batch culture condition [4]. In the field, a 17 months lag time is estimated between a major environmental event (such as hurricane) and before it blooms and releases toxins into the environment and gets into the food chain [83]. During October 1998, a remarkably higher concentration of $G$. toxicus equivalent to 60,463 cells per gram of algae was 
recorded in Mayotte Islands (Comoros, southwest Indian Ocean) following a bloom event. This density is the highest ever recorded in the region and also the highest globally [50]. In a non-bloom event, the cell densities of Gambierdiscus were far less and often non-detectable.

In the western Indian Ocean region, the cell densities of G. toxicus were variable and affected by spatial and temporal variations. In Tanzania, it ranged from 0.0 to 879.5 (Bawe station) cells $\mathrm{g}^{-1}$ wet weight (ww) algae and 0 to 92.6 cells g $^{-1}$ ww seagrass (Mbudya) [66]. The abundances on macroalgae in the Mayotte coral reef complex were reported to range from 0.0 to 2800 cells $\mathrm{g}^{-1}$ [60]. Monitoring of G. toxicus in the locality of Saint Leu (Reunion) since 1993 revealed high variability in population density with an average value of $122 \pm 24$ cells $\mathrm{g}^{-1}$ of algal turf [64]. In the coral reef complex of Mayotte Island (SW Indian ocean), the concentrations of $G$. toxicus ranged from 800-5400 dinoflagellates $\mathrm{g}^{-1}$ of biodeposits in the northeast lagoon. Up to 6000 dinoflagellates $\mathrm{g}^{-1}$ were recorded at Bambo islet in the southeast lagoon. Lower abundances up to $400 \mathrm{~g}^{-1}$ were recorded near the main island shore; seawards on the outer side of the barrier reef; and in luxurious coral areas more exposed to humans. At stations near the main island coast, abundance of G. toxicus was less than 100 cells $\mathrm{g}^{-1}$ [60]. The density of G. toxicus was as low as $0-4$ cells $^{-1}$ of macrolagae in the lagoons of Trou Aux Biches, Mauritius [84].

In the northern part of the Indian ocean, the cell numbers of Gambierdiscus from the central Red Sea, Saudi Arabian coast were $<40$ cells $\mathrm{g}^{-1}$ ww of algae [43,44]. Similarly, F. yasumotoi in Kuwaiti shores had an average cell density of $116.7 \pm 47.5$ cells g $^{-1}$ of ww algae. The high biomass algae (HABs) were estimated through the Ocean Color Modis Algorithm (OC3M), Garver-Siegel-Maritorena Algorithm (GSM), Generalized Inherent Optical Property (GIOP) model [41,45]; these areas when sampled showed the presence of $G$. toxicus in a concentration of $\sim 1000$ cells per liter of seawater [41].

\subsection{Ciguatera Causative Organisms' Substrates and Co-Occurring Species}

Gambierdiscus is an epiphytic benthic dinoflagellate, commonly found on algal turfs, coral rubble, and macroalgae. A study from the Coastal region of Tanzania reported the $G$. toxicus strains along with unknown brown algae mixed with filamentous cyanobacteria species and the red alga Gracillaris sps. [66]. The dinoflagellate was also separated from two species of seagrass namely Thalassia hemprichii and Thalasodendron ciliatum in the same study [66]. The dinoflagellate assemblage observed in coral reefs revealed toxic species including G. toxicus, Prorocentrum spp., and Ostreopsis spp [85]. The co-occurrence of Prorocentrum sps. and Ostreospis sps. with G. toxicus were reported by Grzebyk and his group. In the same study, it was also demonstrated that some microalga stimulated whereas the others subsided the growth of G. toxicus [60]. Benthic thecate dinoflagellates in the sandy ecosystem of La Possession bay (Reunion Islands) were isolated as a complex of five toxic species namely G. yasumotoi, G. toxicus, P. arenarium, P. concavum, and P. lima. At St. Leu and Saline reef, 25 benthic thecate dinoflagellate species coexisted out of which 15 were harmful and are presented in Table 1 [65]. The benthic dinoflagellate of G. toxicus was reported to coexist with Ostreopsis spp., Prorocentrum spp., Coolia monotis, and Amphidinium sp. in the lagoon of Trou Aux Biches, Mauritius [84]. In Mayotte islands, South west of Indian Ocean, G. toxicus were reported along with Prorocentrum spp., Ostreopsis sp., and Amphidinium spp. [50,59]. 
Table 1. Occurrence of Gambierdiscus species in the Indian Ocean region.

\begin{tabular}{|c|c|c|c|c|c|c|c|c|}
\hline Species & Location & $\begin{array}{l}\text { Marginal } \\
\text { Seas }\end{array}$ & $\begin{array}{c}\text { GPS } \\
\text { Coordinates }\end{array}$ & $\begin{array}{l}\text { Sampling } \\
\text { Depth }\end{array}$ & Temperature & Associated Species & Salinities & Reference \\
\hline G. toxicus & Kuwait & $\begin{array}{l}\text { Persian } \\
\text { Gulf }\end{array}$ & $\begin{array}{l}29.3860^{\circ} \mathrm{N} \\
47.7830^{\circ} \mathrm{E}\end{array}$ & & $17.59^{\circ} \mathrm{C}$ & $\begin{array}{c}\text { Karenia selliformis, Karenia } \\
\text { brevis, Prorocentrum lima, } \\
\text { Phalacroma rotundatum, } \\
\text { Tripos muelleri and } \\
\text { Mesodinium rubrum }\end{array}$ & & {$[41,45]$} \\
\hline F. yasumotoi & $\begin{array}{l}\text { Qit'at } \\
\text { Julai'ah, } \\
\text { Kuwait } \\
\text { Semi }\end{array}$ & $\begin{array}{l}\text { Persian } \\
\text { Gulf }\end{array}$ & $\begin{array}{l}28.8370^{\circ} \mathrm{N} \\
48.8160^{\circ} \mathrm{E}\end{array}$ & $1-3 \mathrm{~m}$ & $21-22.3^{\circ} \mathrm{C}$ & $\begin{array}{l}\text { Padina tetrastomatica, } \\
\text { Sargassum oligocystum, } \\
\text { Chaetomorpha sp., }\end{array}$ & $41.2-42.4$ & [42] \\
\hline F. yasumotoi & $\begin{array}{c}\text { protected } \\
\text { shallow } \\
\text { lagoons, } \\
\text { Kuwait }\end{array}$ & $\begin{array}{l}\text { Persian } \\
\text { Gulf }\end{array}$ & $\begin{array}{l}28.8530^{\circ} \mathrm{N} \\
48.8230^{\circ} \mathrm{E}\end{array}$ & $1-3 \mathrm{~m}$ & $23.5-25^{\circ} \mathrm{C}$ & $\begin{array}{c}\text { Amphidinium carterae, } \\
\text { Coolia monotis, Ostreopsis } \\
\text { sps., Prorocentrum } \\
\text { formosum, } P \text {. }\end{array}$ & $41.2-42.4$ & [42] \\
\hline F. yasumotoi & $\begin{array}{l}\text { Southern } \\
\text { coast, } \\
\text { Kuwait }\end{array}$ & $\begin{array}{l}\text { Persian } \\
\text { Gulf }\end{array}$ & $\begin{array}{l}28.8530^{\circ} \mathrm{N} \\
48.8230^{\circ} \mathrm{E}\end{array}$ & $1-3 \mathrm{~m}$ & $23.5-25^{\circ} \mathrm{C}$ & $\begin{array}{l}\text { tsawwassenense, Blixaea } \\
\text { quinquecornis, Adenoides } \\
\text { eludens and Cabra matta }\end{array}$ & $42.1-42.8$ & [42] \\
\hline F. yasumotoi & Jordan & $\begin{array}{l}\text { Gulf of } \\
\text { Aqaba }\end{array}$ & $\begin{array}{c}29.8250^{\circ} \\
\mathrm{N}, 34.8580^{\circ} \mathrm{E}\end{array}$ & $1.5-2 \mathrm{~m}$ & $24-25^{\circ} \mathrm{C}$ & NA & 40.0 & [42] \\
\hline G. belizeanus & Jordan & $\begin{array}{l}\text { Gulf of } \\
\text { Aqaba }\end{array}$ & $\begin{array}{l}29.8250^{\circ} \mathrm{N} \\
34.8580^{\circ} \mathrm{E}\end{array}$ & $1.5-2 \mathrm{~m}$ & $24-25^{\circ} \mathrm{C}$ & NA & 40.0 & [42] \\
\hline G. belizeanus & $\begin{array}{l}\text { Qita Al } \\
\text { Kirsh, } \\
\text { Saudi } \\
\text { Arabia } \\
\text { Sh'ib }\end{array}$ & Red Sea & $\begin{array}{l}22.6058^{\circ} \mathrm{N} \\
39.1980^{\circ} \mathrm{E}\end{array}$ & $0.5-0.6 \mathrm{~m}$ & $29.3^{\circ} \mathrm{C}$ & $\begin{array}{c}\text { Turbinaria decurrens and } \\
\text { Halimeda sp. }\end{array}$ & 38.4 & {$[43,44]$} \\
\hline G. belizeanus & $\begin{array}{l}\text { Nazar, } \\
\text { Saudi } \\
\text { Arabia }\end{array}$ & Red Sea & $\begin{array}{l}22.4916^{\circ} \mathrm{N} \\
38.9722^{\circ} \mathrm{E}\end{array}$ & $0.4 \mathrm{~m}$ & $30.1^{\circ} \mathrm{C}$ & Turbinaria decurrens & 28.9 & {$[43,44]$} \\
\hline G. belizeanus & $\begin{array}{l}\text { Malathu } \\
\text { Reef, Saudi } \\
\text { Arabia }\end{array}$ & Red Sea & $\begin{array}{l}19.8194^{\circ} \mathrm{N} \\
39.9194^{\circ} \mathrm{E}\end{array}$ & $0.5-0.7 \mathrm{~m}$ & NA & Turbinaria decurrens & NA & {$[43,44]$} \\
\hline G. belizeanus & $\begin{array}{l}\text { Marmar } \\
\text { Reef, Saudi } \\
\text { Arabia }\end{array}$ & Red Sea & $\begin{array}{l}19.9038^{\circ} \mathrm{N} \\
39.9947^{\circ} \mathrm{E}\end{array}$ & $0.8 \mathrm{~m}$ & NA & Turbinaria decurrens & NA & {$[43,44]$} \\
\hline G. belizeanus & $\begin{array}{l}\text { Al Fahal, } \\
\text { Saudi } \\
\text { Arabia } \\
\text { Abu }\end{array}$ & Red Sea & $\begin{array}{l}22.5386^{\circ} \mathrm{N} \\
38.9813^{\circ} \mathrm{E}\end{array}$ & $0.6-0.8 \mathrm{~m}$ & $30.4^{\circ} \mathrm{C}$ & $\begin{array}{c}\text { Turbinaria decurrens and } \\
\text { Halimeda sp. }\end{array}$ & 36.0 & {$[43,44]$} \\
\hline G. belizeanus & $\begin{array}{l}\text { Shosha, } \\
\text { Saudi } \\
\text { Arabia }\end{array}$ & Red Sea & $\begin{array}{l}22.3505^{\circ} \mathrm{N} \\
39.2811^{\circ} \mathrm{E}\end{array}$ & $0.5 \mathrm{~m}$ & $29.6^{\circ} \mathrm{C}$ & $\begin{array}{c}\text { Turbinaria decurrens and } \\
\text { Halimeda sp. }\end{array}$ & 38.3 & {$[43,44]$} \\
\hline G. belizeanus & $\begin{array}{l}\text { Um Al } \\
\text { Balam, } \\
\text { Saudi } \\
\text { Arabia }\end{array}$ & Red Sea & $\begin{array}{l}22.4277^{\circ} \mathrm{N} \\
38.9652^{\circ} \mathrm{E}\end{array}$ & $0.4-0.6 \mathrm{~m}$ & $29.3^{\circ} \mathrm{C}$ & $\begin{array}{c}\text { Turbinaria decurrens and } \\
\text { Halimeda sp. }\end{array}$ & 36.1 & {$[43,44]$} \\
\hline G. belizeanus & $\begin{array}{l}\text { Um Al } \\
\text { Kiethl, } \\
\text { Saudi } \\
\text { Arabia }\end{array}$ & Red Sea, & $\begin{array}{l}22.3197^{\circ} \mathrm{N} \\
39.0541^{\circ} \mathrm{E}\end{array}$ & $0.5 \mathrm{~m}$ & $29.5^{\circ} \mathrm{C}$ & $\begin{array}{c}\text { Turbinaria decurrens and } \\
\text { Halimeda sp. }\end{array}$ & 28.9 & {$[43,44]$} \\
\hline G. belizeanus & $\begin{array}{l}\text { Coast } \\
\text { Guard } \\
\text { Reef, Saudi } \\
\text { Arabia }\end{array}$ & Red Sea & $\begin{array}{l}20.3950^{\circ} \mathrm{N} \\
40.3580^{\circ} \mathrm{E}\end{array}$ & $0.5 \mathrm{~m}$ & NA & $\begin{array}{c}\text { Turbinaria decurrens and } \\
\text { Halimeda sp. }\end{array}$ & NA & {$[43,44]$} \\
\hline G. belizeanus & $\begin{array}{l}\text { Mangrove } \\
\text { Reef, Saudi } \\
\text { Arabia }\end{array}$ & Red Sea & $\begin{array}{l}20.4400^{\circ} \mathrm{N} \\
40.2911^{\circ} \mathrm{E}\end{array}$ & $0.4 \mathrm{~m}$ & NA & Turbinaria decurrens & NA & {$[43,44]$} \\
\hline G. toxicus & $\begin{array}{l}\text { Sindh } \\
\text { Coast, } \\
\text { Pakistan }\end{array}$ & $\begin{array}{l}\text { Manora } \\
\text { Channel; } \\
\text { Chuma } \\
\text { Island }\end{array}$ & $\begin{array}{l}24.8091^{\circ} \mathrm{N} \\
66.9908^{\circ} \mathrm{E}\end{array}$ & $1.0 \mathrm{~m}$ & $32^{\circ} \mathrm{C}$ & & & [69] \\
\hline G. toxicus & $\begin{array}{l}\text { Balochistan } \\
\text { Coast, } \\
\text { Pakistan }\end{array}$ & $\begin{array}{l}\text { Manora } \\
\text { Channel; } \\
\text { Chuma } \\
\text { Island }\end{array}$ & $\begin{array}{l}24.7861^{\circ} \mathrm{N} \\
66.4516^{\circ} \mathrm{E}\end{array}$ & $1.0 \mathrm{~m}$ & $28^{\circ} \mathrm{C}$ & & & [69] \\
\hline
\end{tabular}


Table 1. Cont.

\begin{tabular}{|c|c|c|c|c|c|c|c|c|}
\hline Species & Location & $\begin{array}{l}\text { Marginal } \\
\text { Seas }\end{array}$ & $\begin{array}{c}\text { GPS } \\
\text { Coordinates }\end{array}$ & $\begin{array}{l}\text { Sampling } \\
\text { Depth }\end{array}$ & Temperature & Associated Species & Salinities & Reference \\
\hline G. belizeanus & $\begin{array}{l}\text { Sindh } \\
\text { Coast, } \\
\text { Pakistan }\end{array}$ & $\begin{array}{l}\text { Manora } \\
\text { Channel; } \\
\text { Chuma } \\
\text { Island } \\
\text { Manora }\end{array}$ & $\begin{array}{l}24.8091^{\circ} \mathrm{N} \\
66.9908^{\circ} \mathrm{E}\end{array}$ & $1.0 \mathrm{~m}$ & $32{ }^{\circ} \mathrm{C}$ & & & [69] \\
\hline G. belizeanus & $\begin{array}{l}\text { Balochistan } \\
\text { Coast }\end{array}$ & $\begin{array}{l}\text { Channel; } \\
\text { Chuma } \\
\text { Island, } \\
\text { Pakistan }\end{array}$ & $\begin{array}{l}24.7861^{\circ} \mathrm{N} \\
66.4516^{\circ} \mathrm{E}\end{array}$ & $1.0 \mathrm{~m}$ & $28^{\circ} \mathrm{C}$ & & & [69] \\
\hline G. australes & $\begin{array}{l}\text { Sindh } \\
\text { Coast }\end{array}$ & $\begin{array}{l}\text { Manora } \\
\text { Channel; } \\
\text { Chuma } \\
\text { Island, } \\
\text { Pakistan }\end{array}$ & $\begin{array}{l}24.8091^{\circ} \mathrm{N} \\
66.9908^{\circ} \mathrm{E}\end{array}$ & $1.0 \mathrm{~m}$ & $32{ }^{\circ} \mathrm{C}$ & & & [69] \\
\hline G. australes & $\begin{array}{l}\text { Balochistan } \\
\text { Coast }\end{array}$ & $\begin{array}{l}\text { Manora } \\
\text { Channel; } \\
\text { Chuma } \\
\text { Island, } \\
\text { Pakistan }\end{array}$ & $\begin{array}{l}24.7861^{\circ} \mathrm{N} \\
66.4516^{\circ} \mathrm{E}\end{array}$ & $1.0 \mathrm{~m}$ & $28^{\circ} \mathrm{C}$ & & & [69] \\
\hline $\begin{array}{c}\text { G. } \\
\text { polynesiensis }\end{array}$ & $\begin{array}{l}\text { Sindh } \\
\text { Coast }\end{array}$ & $\begin{array}{l}\text { Manora } \\
\text { Channel; } \\
\text { Chuma } \\
\text { Island, } \\
\text { Pakistan }\end{array}$ & $\begin{array}{l}24.8091^{\circ} \mathrm{N} \\
66.9908^{\circ} \mathrm{E}\end{array}$ & $1.0 \mathrm{~m}$ & $32{ }^{\circ} \mathrm{C}$ & & & [69] \\
\hline $\begin{array}{c}G . \\
\text { polynesiensis }\end{array}$ & $\begin{array}{l}\text { Balochistan } \\
\text { Coast }\end{array}$ & $\begin{array}{l}\text { Manora } \\
\text { Channel; } \\
\text { Chuma } \\
\text { Island, } \\
\text { Pakistan }\end{array}$ & $\begin{array}{l}24.7861^{\circ} \mathrm{N} \\
66.4516^{\circ} \mathrm{E}\end{array}$ & $1.0 \mathrm{~m}$ & $28^{\circ} \mathrm{C}$ & & & [69] \\
\hline F. yasumotoi & $\begin{array}{l}\text { Sindh } \\
\text { Coast }\end{array}$ & $\begin{array}{l}\text { Manora } \\
\text { Channel; } \\
\text { Chuma } \\
\text { Island, } \\
\text { Pakistan }\end{array}$ & $\begin{array}{l}24.8091^{\circ} \mathrm{N} \\
66.9908^{\circ} \mathrm{E}\end{array}$ & $1.0 \mathrm{~m}$ & $32{ }^{\circ} \mathrm{C}$ & & & [69] \\
\hline F. yasumotoi & $\begin{array}{l}\text { Balochistan } \\
\text { Coast }\end{array}$ & $\begin{array}{l}\text { Manora } \\
\text { Channel; } \\
\text { Chuma } \\
\text { Island, } \\
\text { Pakistan }\end{array}$ & $\begin{array}{l}24.7861^{\circ} \mathrm{N} \\
66.4516^{\circ} \mathrm{E}\end{array}$ & $1.0 \mathrm{~m}$ & $28^{\circ} \mathrm{C}$ & & & [69] \\
\hline $\begin{array}{l}\text { Gambierdiscus } \\
\text { sp. }\end{array}$ & $\begin{array}{l}\text { Bay of } \\
\text { Bengal }\end{array}$ & India & & & & & & [71] \\
\hline G. toxicus & $\begin{array}{l}\text { Mbudya } \\
\text { Island, } \\
\text { Dar es } \\
\text { Salaam }\end{array}$ & Tanzania & & $5.0 \mathrm{~m}$ & $25.5-31.0^{\circ} \mathrm{C}$ & $\begin{array}{l}\text { Seagrass- } \\
\text { Thalassodendron } \\
\text { ciliatum }\end{array}$ & $35-37$ & [66] \\
\hline G. toxicus & $\begin{array}{l}\text { Bawe } \\
\text { Island, } \\
\text { Zanzibar }\end{array}$ & Tanzania & & $10.0 \mathrm{~m}$ & $26.0-29.0^{\circ} \mathrm{C}$ & $\begin{array}{l}\text { Unknown brown algae } \\
\text { mixed with Filamentous } \\
\text { cyanobacteria }\end{array}$ & $34-36$ & [66] \\
\hline G. toxicus & $\begin{array}{l}\text { Makoba, } \\
\text { Zanzibar }\end{array}$ & Tanzania & & $10.0 \mathrm{~m}$ & $26.0^{\circ} \mathrm{C}$ & Gracilaria sp. & $35-43$ & [66] \\
\hline G. toxicus & $\begin{array}{l}\text { Longoni } \\
\text { Bay } \\
\text { (Stations } \\
1 \text { and } 2 \text { ) }\end{array}$ & $\begin{array}{l}\text { Mayotte, } \\
\text { Mozam- } \\
\text { bique }\end{array}$ & $\begin{array}{l}12.8333^{\circ} \mathrm{N} \\
45.1666^{\circ} \mathrm{E}\end{array}$ & & & & & [60] \\
\hline G. toxicus & $\begin{array}{l}1 \text { and 2) } \\
\text { Surprise } \\
\text { Reef } \\
\text { (Station } \\
3 \text { ) }\end{array}$ & $\begin{array}{l}\text { Mayotte, } \\
\text { Mozam- } \\
\text { bique }\end{array}$ & $\begin{array}{l}12.8333^{\circ} \mathrm{N} \\
45.1666^{\circ} \mathrm{E}\end{array}$ & $\begin{array}{l}\text { Between the } \\
\text { depth of the } \\
\text { lower } \\
\text { intertidal level } \\
\text { and } 30 \mathrm{~m}\end{array}$ & $26-31^{\circ} \mathrm{C}$ & $\begin{array}{c}\text { Ostreopsis lenticularis; } O . \\
\text { lenticularis; Prorocentrum } \\
\text { lima and } 4 \text { new species of } \\
\text { Prorocentrum }\end{array}$ & $35-37$ & [60] \\
\hline
\end{tabular}


Table 1. Cont.

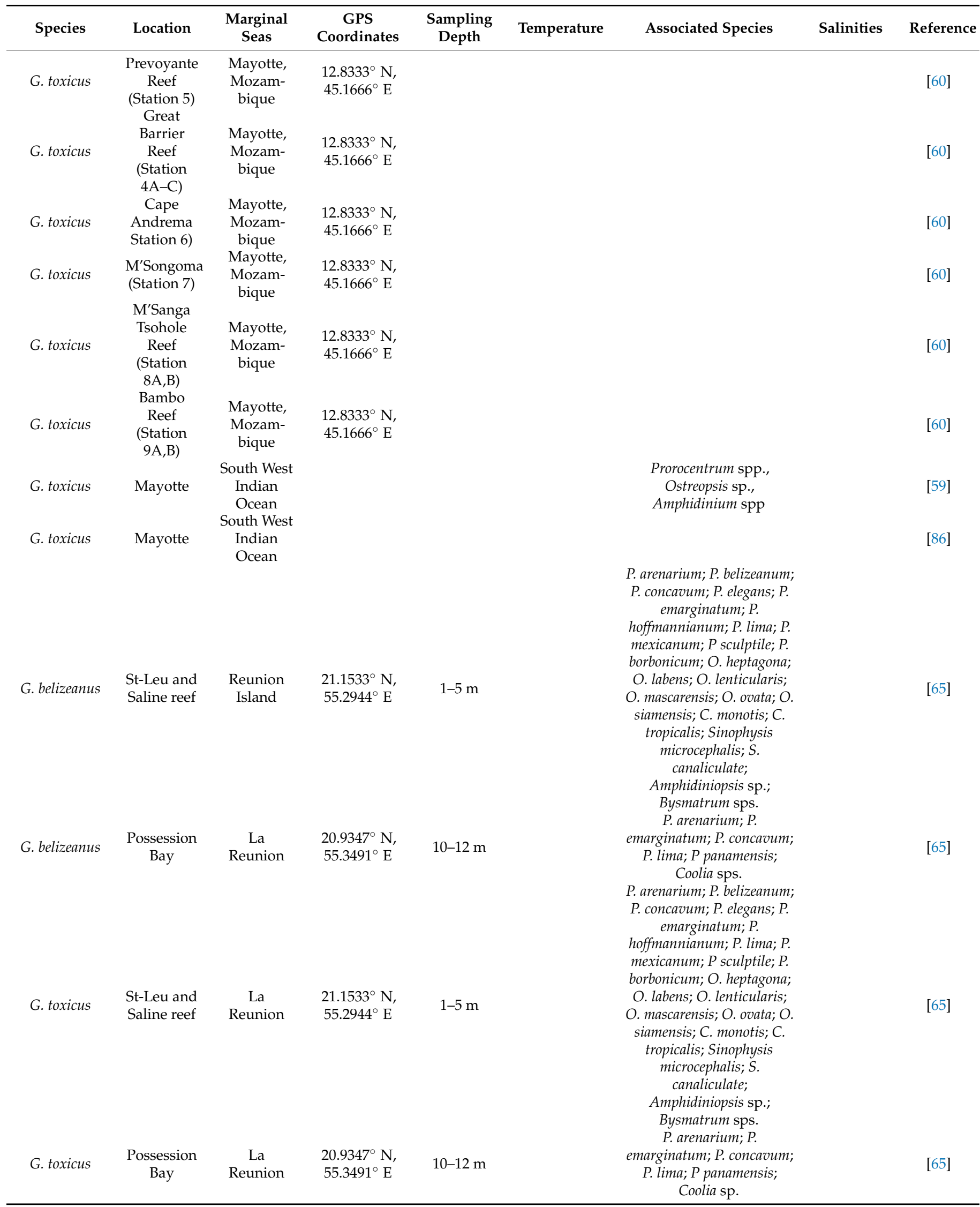


Table 1. Cont.

\begin{tabular}{|c|c|c|c|c|c|c|c|c|}
\hline Species & Location & $\begin{array}{l}\text { Marginal } \\
\text { Seas }\end{array}$ & $\begin{array}{c}\text { GPS } \\
\text { Coordinates }\end{array}$ & $\begin{array}{l}\text { Sampling } \\
\text { Depth }\end{array}$ & Temperature & Associated Species & Salinities & Reference \\
\hline $\begin{array}{c}\text { F. ya- } \\
\text { sumotoi }\end{array}$ & $\begin{array}{l}\text { St-Leu and } \\
\text { Saline reef }\end{array}$ & La Reunion & $\begin{array}{l}21.1533^{\circ} \mathrm{N} \\
55.2944^{\circ} \mathrm{E}\end{array}$ & $1-5 \mathrm{~m}$ & \multirow{2}{*}{\multicolumn{2}{|c|}{$\begin{array}{l}\text { P. arenarium; P. belizeanum; } \\
\text { P. concavum; P. elegans; } P \text {. } \\
\text { emarginatum; } P \text {. } \\
\text { hoffmannianum; P. lima; } P \text {. } \\
\text { mexicanum; } P \text { sculptile; } P \text {. } \\
\text { borbonicum; O. heptagona; } \\
\text { O. labens; O. lenticularis; } \\
\text { O. mascarensis; O. ovata; } O \text {. } \\
\text { siamensis; C. monotis; } C \text {. } \\
\text { tropicalis; Sinophysis } \\
\text { microcephalis; } S . \\
\text { canaliculate; } \\
\text { Amphidiniopsis sp.; } \\
\text { Bysmatrum sp. } \\
\text { P. arenarium; } P . \\
\text { emarginatum; P. concavum; } \\
\text { P. lima; P panamensis; } \\
\text { Coolia sp. }\end{array}$}} & & [65] \\
\hline $\begin{array}{c}\text { F. ya- } \\
\text { sumotoi }\end{array}$ & $\begin{array}{c}\text { Possession } \\
\text { Bay }\end{array}$ & La Reunioin & $\begin{array}{c}20.9347^{\circ} \mathrm{N} \\
55.3491^{\circ} \mathrm{E}\end{array}$ & $10-12 \mathrm{~m}$ & & & & [65] \\
\hline $\begin{array}{c}\text { G. } \\
\text { toxicus }\end{array}$ & $\begin{array}{l}\text { Reunioin } \\
\text { Islands }\end{array}$ & Mauritius & & & & $\begin{array}{c}\text { Prorocentrum sp.; } \\
\text { Ostreopsis sp. }\end{array}$ & & [64] \\
\hline \multicolumn{2}{|c|}{ Gambierdiscus Mangalore } & India & $\begin{array}{l}12.91777^{\circ} \mathrm{N} \\
74.79162^{\circ} \mathrm{E}\end{array}$ & & & & & [72-74] \\
\hline
\end{tabular}

NA-Not available.

In the reports of Catania and her team [43,44], the Red Sea strains of G. belizeanus were associated with the macroalgae species of Turbinaria decurrens and Halimeda sps. Information on macroalgal species associated with G. belizeanus isolated from the Gulf of Aqaba is unavailable [42]. In the case of G. yasumotoi, the macroalgal substrates were identified as Padina tetrastomatica and Sargassum oligocystum in winter (November 2012) collections whereas in summers (March 2013) they occurred along with Chaetomorpha sp. The group also reported the co-occurrence of macroalgal species of Amphidinium carterae, Coolia monotis, Ostreopsis sps., Prorocentrum formosum, P. tsawwassenense, Peridinium quinquecorne, Adenoides eludens, and Cabra matta [42]. Moderate Resolution Imaging Spectroradiometer (MODIS) and Medium Resolution Imaging Spectrometer (MERIS) data and its various operational algorithms such as OC3M-547, GSM, GIOP, and other bio-optical methods developed by Uddin and coworkers $[41,45,54]$, revealed algal blooms; during field verification, it was observed that G. toxicus was also part of the algal bloom on 21 December 2009. Samples collected from location $29.38694^{\circ} \mathrm{N}, 47.78389^{\circ} \mathrm{E}$ had over 1000 cells/L. Other algae identified in the field samples were G. toxicus, Karenia selliformii, K. brevis, P. lima, Dinophysis rotundata, Ceratium tripos, and Myrionecta rubrum.

Recent deliberations by the experts in an international meeting in Rome on ciguatera poisoning recommended the next generation sequencing (NGS) to mass-amplify specific gene sequences from sediment samples to characterize all species or specific taxa present in the sample [8]. This approach allows better identification and resolution of microbial community composition than the conventional morphological and molecular methodologies. The information on ciguatera from the Indian Ocean region is still in its initial phase. This approach would provide comprehensive information on ciguatera diversity and its interaction with associated communities as reported elsewhere [87-91].

\subsection{Ciguatera Causative Organism's Morphology and Phylogeny}

A variety of techniques are known to be used for ciguatera identification at a particular site. These include light microscopy (LM), scanning electron microscopy (SEM), DNA sequencing, restriction fragment length polymorphism (RFLP), quantitative polymerase chain reaction (qPCR), and metabarcoding [8,87]. While performing the morphological identification, the taxonomic key reported earlier [25] was followed by the research groups 
to confirm the species of Gambierdiscus found in the region. Earlier in the 1990s, SEM was used for the identification of G. toxicus from Reunion Islands [64]. SEM was also used recently to identify the species of G. toxicus; G. belizeanus; G. polynesiensis; G. australes; and F. yasumotoi [69]. The morphological identification of Gulf strains of G. yasumotoi and G. belizeanus was based on both LM and SEM [42-44]. A description of morphological parameters of strains present in the Indian Ocean region is given in Table 2.

Table 2. Morphological features of Gambierdiscus species found in the Indian Ocean region.

\begin{tabular}{|c|c|c|c|c|c|}
\hline & F. yasumotoi [12] & G. belizeanus [92] & G. toxicus [93] & $\begin{array}{c}\text { G. polynesiensis } \\
\text { [94] }\end{array}$ & G. australes [94] \\
\hline Cell depth & 54 to $73 \mu \mathrm{m}$ & 59 to $73 \mu \mathrm{m}$ & 71 to $96 \mu \mathrm{m}$ & 65 to $87 \mu \mathrm{m}$ & 60 to $82 \mu \mathrm{m}$ \\
\hline Cell width & 46 to $61 \mu \mathrm{m}$ & 60 to $73 \mu \mathrm{m}$ & 45 to $140 \mu \mathrm{m}$ & 64 to $75 \mu \mathrm{m}$ & 65 to $85 \mu \mathrm{m}$ \\
\hline Cell length & 49 to $70 \mu \mathrm{m}$ & 47 to $51 \mu \mathrm{m}$ & 50 to $150 \mu \mathrm{m}$ & 57 to $75 \mu \mathrm{m}$ & 76 to $93 \mu \mathrm{m}$ \\
\hline Cell shape & Globular & Round & Rounded/ellipsoid & Rounded/ellipsoid & Rounded/ellipsoid \\
\hline Apical pore & Fish hook-shaped & Fish hook-shaped & Fish hook shaped & Fish hook shaped & Fish hook shaped \\
\hline Thecal surface & & $\begin{array}{c}\text { Moderately } \\
\text { aerated }\end{array}$ & & Smooth & Smooth \\
\hline Apical plate & $\begin{array}{l}\text { elongated and } \\
\text { teardrop-shaped }\end{array}$ & Ellipsoid & Ellipsoid & $\begin{array}{l}\text { large triangular } \\
\text { apical pore plate }\end{array}$ & $\begin{array}{l}\text { broad ellipsoid } \\
\text { apical pore plate }\end{array}$ \\
\hline Hypotheca & & Truncate-conical & & & $\begin{array}{l}\text { Eight hypothecal } \\
\text { plates }\end{array}$ \\
\hline $\begin{array}{l}\text { Epitheca } \\
\text { Sulcus }\end{array}$ & & dome-shaped & $\begin{array}{c}\text { Ellipsoid } \\
\text { Short }\end{array}$ & & Eleven plates \\
\hline Chloroplast & $\begin{array}{c}\text { elongated } \\
\text { golden-brown } \\
\text { chloroplasts were } \\
\text { peripherally } \\
\text { arranged }\end{array}$ & $\begin{array}{l}\text { golden-brown } \\
\text { drop-shaped } \\
\text { chloroplasts } \\
\text { radiating from the } \\
\text { cell centre }\end{array}$ & & & \\
\hline Nucleus & $\begin{array}{l}\text { large, oblong and } \\
\text { located in the right } \\
\text { posterior }\end{array}$ & $\begin{array}{c}\text { large, oblong and } \\
\text { crescent-shaped, } \\
\text { and located in the } \\
\text { right posterior cell } \\
\text { part }\end{array}$ & & & $\begin{array}{c}\text { Posteriorly } \\
\text { situated nucleus }\end{array}$ \\
\hline Cingulum & & $\begin{array}{l}\text { deeply excavated, } \\
\text { descending, }\end{array}$ & $\begin{array}{l}\text { Ascending narrow } \\
\text { cingulum }\end{array}$ & & Narrow and deep \\
\hline Plate Formulae & & & $\begin{array}{c}\text { Po, } 3^{\prime}, 7^{\prime \prime}, 6 \mathrm{C}, 8 \mathrm{~S} \\
5^{\prime \prime \prime}, 1 \mathrm{p}, 2^{\prime \prime \prime}\end{array}$ & $\begin{array}{c}\text { Po, } 3^{\prime}, 7^{\prime \prime}, 6 \mathrm{C}, 8 \mathrm{~S}, \\
5^{\prime \prime \prime}, 1 \mathrm{p} \text { et } 2^{\prime \prime \prime}\end{array}$ & $\begin{array}{c}\text { Po, } 3^{\prime}, 7^{\prime \prime}, 6 \mathrm{C}, 8 \mathrm{~S} \\
5^{\prime \prime \prime}, 1 \mathrm{p} \text { et } 2^{\prime \prime \prime}\end{array}$ \\
\hline Studied by & {$[42,69]$} & {$[42-44,69]$} & {$[66,69]$} & & {$[69]$} \\
\hline
\end{tabular}

Owing to the plasticity of morphological characteristics of some species of Gambierdiscus, the identification can be ambiguous if only morphology is used. Currently, molecular techniques are gaining popularity for the confirmation of species identified through microscopic methods. The only report on molecular identification of Gambierdiscus in the Arabian Gulf was by Catania [43] and her team [44]. The group amplified an 850 bp hypervariable (D8-D10) region of the larger subunit (LSU) of rRNA using primers FD8 and RB [11,25] through Sanger sequencing. A query of assembled nucleotides on the National Centre for Biotechnology and Informatics (NCBI) through the basic local alignment search tool (BLAST) resulted in a 100\% match with G. belizeanus. Their (KY782637-KY782645) strains depicted a close resemblance with the Caribbean strain of $G$. belizeanus. The group also reported a 116 bp deletion at 493-609 position in one of the isolates, probably imparting the distinctiveness from other species of Gambierdiscus $[95,96]$.

Phylogenetic analysis (methodology described in the Supplementary file) based on multiple alignments (Clustal2.1) of all the available DNA sequences $(n=345)$ of the D8-D10 region of the large sub unit (LSU) of ribosomal RNA (rRNA) [9,11,14,15,25,26,40,43,44,89,96-114] distributes the Gambierdiscus genus into five major clades (clade I-V) as seen in Figure 8. Clade II and III exclusively contain the species G. australes (Atlantic and Pacific strains) and G. excentricus (Atlantic strains), respectively. Clade I encompass all of the G. carribaeus (Atlantic and Pacific 
strains) and newly discovered G. carpenteri (Atlantic and Pacific strains) as well as G. jejuensis (Pacific strains). Clade IV and V are more diverse. Of this, G. belizeanus (Red Sea, Saudi Arabia; Charco Azul, El Hierro, Spain; St. Barthelemy Island, Caribbean Sea) appears as a distinct branch in Clade $\mathrm{V}$ with the closest genetic relationship with G. honu (Kermadec Islands, Australia). The yet unclassified Gambierdiscus ribotype 1 follows the same lineage as of G. belizeanus. Other species in close proximity were G. balechii (Celebes Sea, Pacific Ocean; Phuket Islands, Indonesia, North Pacific), G. lapillus (Great Barrier Reef, Australia, Pacific Ocean; Cook Islands, Rarotonga, North Atlantic), G. cheloniae (Rarotonga, Cook Islands, North Atlantic), G. scabrous (Japan, South China Sea, North Pacific), and G. pacificus (Balearic Islands, Spain, North Atlantic; Cook Islands, Rarotonga, North Atlantic; Marshall Islands, Micronesia, South Pacific). The G. toxicus (Indian Ocean) strains are also in the same clade.

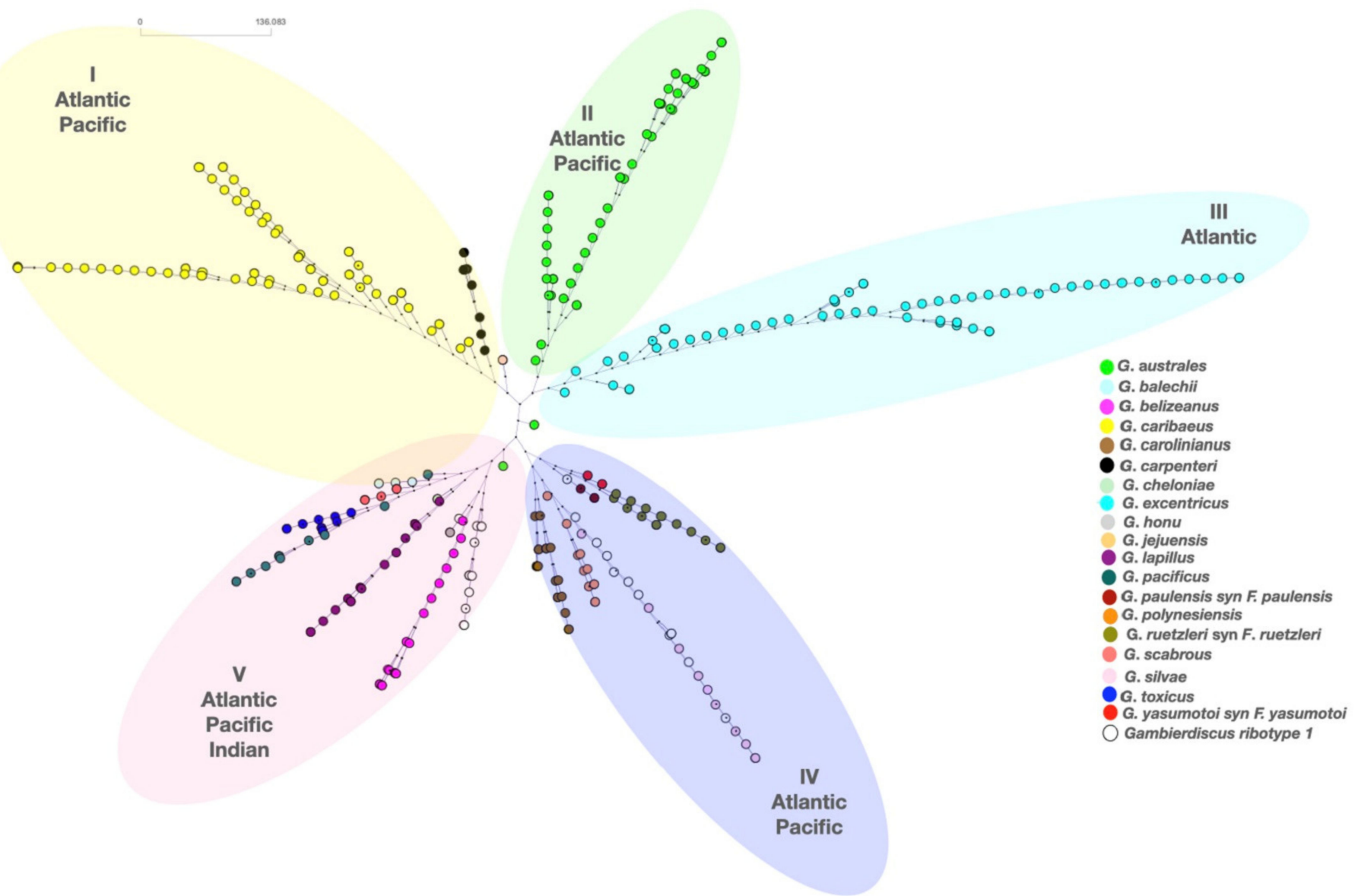

Figure 8. Phylogenetic associations of globally distributed Gambierdiscus and Fukuyoa species. A split decomposition algorithm was applied on multiple aligned D8-D10 sequences of LSU of available Gambierdiscus species (downloaded from NCBI) and aligned through ClustalW2.

Molecular identification of F. yasumotoi on the Kuwaiti shore waters and Gulf of Aqaba have not yet been performed. Similarly, G. australes, G. toxicus, and G. polynesiensis from Manora Channel, Indian Ocean region are pending molecular identification. F. yasumotoi isolated from the Pacific [11] and Atlantic region fall in the same branch as that of F. reutzleri (North Atlantic and North Pacific Ocean) and F. paulensis (North Atlantic and South Pacific Oceans). All the F. yasumotoi strains discovered so far showed a similarity of $\sim 80 \%$ with the unique ribotype A213 [11,97]. Other species in the same clade are that of G. carolinianus (Bermuda, North Atlantic; Bahamas, North Atlantic; Cancun, Mexico, North Atlantic; Caribbean Sea, North Atlantic), G. silvae (Canary Island, North Atlantic), G. polynesiensis (Cook Islands, Rarotonga, North Atlantic; Pacific Ocean), and unclassified Gambierdiscus ribotypes (Curlew Cay, Belize, North Atlantic). 
More diversity in clade IV and V including the species discovered from Indian Ocean may be due to a smaller number of samples owing to the recent discovery of these species. The presence of unclassified Fukuyoa sps. and Gambierdiscus ribotypes in these clades is suggestive of unexplored novel species. In 2016, at the 32nd session of the Codex Committee on Fisheries and Fishery Products, the Pacific Nations raised CP as an issue that is increasingly affecting the tropical and subtropical regions of the Pacific Ocean, Indian Ocean and the Caribbean Sea between the latitudes of $35^{\circ} \mathrm{N}$ and $35^{\circ} \mathrm{S}$ [8]. The strains found in the Indian Ocean and their adjoining seas lie near the strains commonly found in these high-risk regions. Probably, the interconnection between the water bodies and the ballast water is the major source of ciguatera infiltration in the Indian Ocean waters. Given this, extensive monitoring and risk management program should be developed for the unexplored regions and specifically in the Middle East region.

\subsection{Vectors of Ciguatera in Indian Ocean Region}

The $\mathrm{CP}$ is recognized as a tropical disease but the existence of ciguatoxic fishes is reported globally due to international seafood trade and shipment [115]. According to a recent FAO/WHO report, globally 425 species of fish, especially those inhabiting the coral reefs, have been associated with CP [8]. The most significant toxic fish have been made by barracuda (Sphyraenidae), amberjack (Seriola), grouper (Serranidae), snapper (Lutjanidae), po'ou (Labridae spp.), jack (Carangidae spp.), trevally (Caranx spp.), wrasse (Labridae spp.), surgeonfish (Acanthuridae spp.), moray eel (Muraenidae spp.), roi (Cephalopholis spp.), parrotfish (Scaridae spp.), etc.

The $\mathrm{CP}$ cases have mainly been reported in the southwestern region encompassing Comoros, Mayotte, La Reunion, Mauritius, Rodrigues, and Seychelles. The reports from the Northern part of the Indian ocean have been scarce and involve cases in Mangalore and Trivandrum on the southern Indian coast and an outbreak resulting in one fatality from Pakistan, but the fish species involved and their origin could not be confirmed $[74,75,116,117]$. Few cases of CFP were also reported in Thailand after eating ocean fish particularly sea bass and red snapper $[118,119]$. One CP record from Egypt exists [120]. There has been no event recorded in the Northern part of the Arabian sea, in countries bordering the Gulf region or the Red Sea. The very first incidence of ciguatera poisoning in the Indian Ocean region was reported in 1993 from Manakara and Madagascar where about 500 persons were infected after consuming ciguatoxic shark. The fatality rate of this outbreak was 20\% [121]. In Trivandrum, India, an autochthonous outbreak was reported in 2015 and 2016, due to which nearly 200 workers of a fish factory contracted CFP after eating heads of Red Snappers [75]. In 2013, also severe food poisoning events were witnessed after consuming a bull shark (Carcharhinus leucas), resulting in the deaths of 11 people in Madagascar in 2013 [78]. Fish originating from the Indian ocean region were implicated in ciguatera poisoning in other part of the world. The rapid alert system for food and feed (RASFF) created in 1979 by the European Union (EU) successively warned in 2012, 2015, and 2016 about ciguatoxic fishes originating from India and Sri Lanka (RASFF No. 2012-1602, 2015-0088, 2016-0932, 2016-1152, 2016-1155, 2017-1112). In 2016, scientists and regional public health authorities warned the population in Southwest India about the CFP risk caused by the consumption of Red Snappers [122], yet snappers from India were imported by France and distributed to Germany (RASFF No. 2016-0932). The French Poison Control center network reported from 2012 to 2019, 17 events with fish caught from the Indian Ocean. In a recent report from Germany, the rarely occurring CP outbreaks were reported between 2012-2017, the main reason identified as imports of snappers (Lutjanidae) from the Indian Ocean region mainly from countries like India, Indonesia, and Vietnam. The author emphasized that fishes from the Indian Ocean can cause ciguatera, which has been poorly documented [81].

Although incidences of $\mathrm{CP}$ have not been reported from the Arabian Gulf yet, however, the fishes such as grouper, snapper, emperors, barracuda, jacks, trevallies, kingfish, and tuna form the part of common catch by local fishermen in Arabian Gulf [123]. The database search in Fish base returned a minimum of 13 (Iraq) to a maximum of 60 (Oman) fishes 
associated with CP elsewhere in the world [124] (Figure 9). An increase in ciguatera causative organism could have a major impact on food safety and food security given that those species are highly consumed in the region. The suspicion of a high number of unreported cases is warranted given that ciguatera is difficult to diagnose and not subject to mandatory reporting [125].

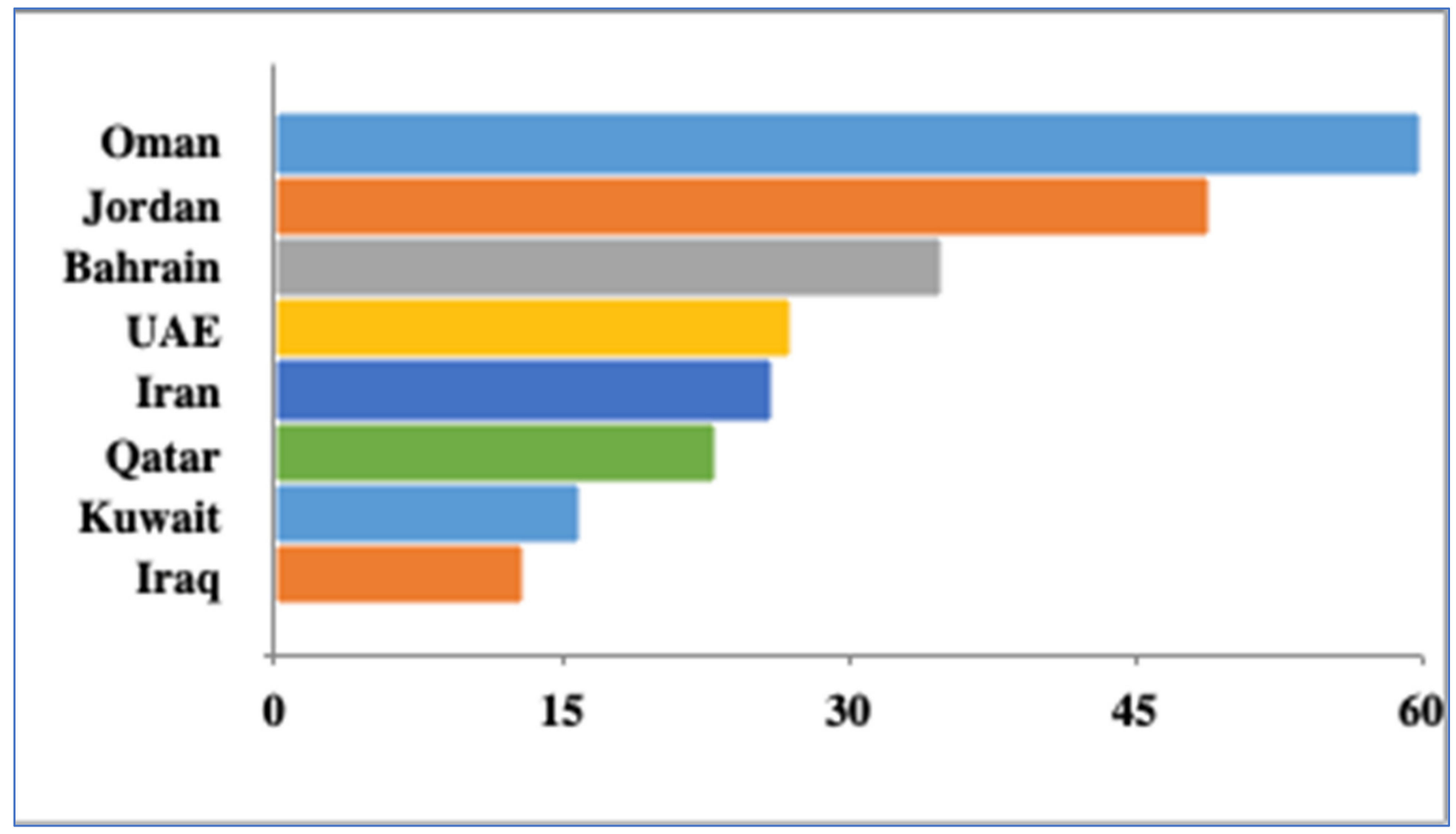

Figure 9. Number of Fishes associated with CP in Persian Gulf Countries as per the records of Fish Base (accessed on 1 June 2021).

The Persian Gulf water is the only source of freshwater through seawater desalination to most Gulf countries and an important source of seafood for the region. Previous reports on incidences of harmful algal blooms on drinking water and food safety and of massive fish kills in the region further creates awareness and interest to investigate the occurrence of ciguatera causative organisms and associated toxins, as well as their health implications in the Gulf countries [58].

\subsection{Ciguatera Monitoring in Indian Ocean Region}

Risk assessment and monitoring of Ciguatera in the Indian Ocean region is largely lacking with only a couple of previous attempts from la Reunion [47] and Mayotte Island [61]. In 2014, an algal bloom monitoring system was developed for Kuwait's coastal waters. The OC3M (Aqua-MODIS) and OC4E (ENVISAT-MERIS) algorithms most accurately measured chlorophyll-a concentrations in Kuwait bay. Due to the poor temporal resolution and the decommissioning of ENVISAT-MERIS, Aqua-MODIS data was used for continuous observation. Additionally, algorithms such as Generalized Inherent Optical Properties (GIOP), Garver, Siegel, Maritorena Model, OC2, MODIS fluorescence line height, and the MERIS-based NIR-Red algorithms were attempted, which have a lower accuracy when compared to the OC3M algorithm. The OC3M detected the most reported in-situ algal bloom events (19/50) and most accurately measured chlorophyll-a concentration (RMS: 2.42, RMSE: 4.11, Mean Bias: 54.2\%). The Aqua-MODIS OC3M was selected as the preferred algorithm to monitor chlorophyll-a concentration and to detect algal blooms in Kuwait bay and surrounding waters [41,45]. Data variables such as sea surface temperature, OC3M, distance to aquaculture, Garver-Siegel-Maritorena (GSM), generalized 
inherent optical properties (GIOP), euphotic depth, Secchi disk depth, distance to shore, precipitation, photosynthetically active radiation (PAR), distance to the river, bathymetry, colored dissolved organic matter, wind direction, speed, and precipitation, etc. were also estimated through a multivariate regression model, a hybrid multivariate regression model, an artificial neural network model, and a hybrid artificial neural network model by Uddin and his group [41,45].

A synergistic model was created that combines the GIS-imaging, the different estimates of environmental parameters, and in situ monitoring with traditional toxin analytical methods $[41,54]$. Sampling epiphytic substrates and analyzing samples using traditional optical microscopy will provide very useful and immediate information and developing ciguatera early warning systems in the region. The molecular methods of qPCR and metabarcoding can be useful but only as a complement not as the basic methodology to estimate Gambierdiscus abundance.

\section{Conclusions}

Ciguatera research in the Indian Ocean region is limited and fragmented. Although, there are reported cases of $\mathrm{CP}$ in the region. The most significant toxic fish in the region were barracuda (Sphyraenidae), amberjack (Seriola), grouper (Serranidae), snapper (Lutjanidae), po'ou (Labridae spp.), jack (Carangidae spp.), trevally (Caranx spp.), wrasse (Labridae spp.), surgeonfish (Acanthuridae spp.), moray eel (Muraenidae spp.), roi (Cephalopholis spp.), parrotfish (Scaridae spp.), seabass, shark, and red snapper to name a few. Since the region exports fisheries, some of the fish originating from India and Sri Lanka in 2012, 2015, and 2016 were implicated in ciguatera poisoning in Europe.

A comprehensive survey of algal substrates in the region complemented with high throughput metabarcoding would provide insights into novel and undiscovered contributors of ciguatera. Although less in numbers, the presence of ciguatera causative organisms in the region cannot be ignored and their interaction with substrate and other microbial species is worthy of further investigation. The development of an early warning system for HABs is very much the need of the hour.

Supplementary Materials: The following are available online at https: / www.mdpi.com/article / 10.3390 / toxins13080525/s1, Metadata and percent identity matrix of 345 Gambierdiscus sequences downloaded from NCBI and aligned using Clustal W2.1.

Author Contributions: Conceptualization, S.U., N.H.; methodology, N.H., S.U.; software, N.H., M.F.; resources, S.U., N.H., M.-Y.D.B., M.F.; data curation, S.U., M.F.; writing —original draft preparation, N.H., S.U.; writing—review and editing, N.H., S.U., M.-Y.D.B.; visualization, M.F., M.-Y.D.B. All authors have read and agreed to the published version of the manuscript.

Funding: This research received no external funding.

Institutional Review Board Statement: Not applicable, since this study does not involve humans or animals.

Informed Consent Statement: Not applicable as no human subject was involved.

Data Availability Statement: Data provided as Supplementary file and within manuscript itself.

Acknowledgments: Authors will like to thank the Kuwait Institute for Scientific Research for supporting this study.

Conflicts of Interest: The authors declare no conflict of interest. The Kuwait Institute for Scientific Research has no role in the design of the study; in the collection, analyses, or interpretation of data; in the writing of the manuscript, or in the decision to publish the results.

\section{References}

1. Senthilkumaran, S.; Meenakshisundaram, R.; Michaels, A.; Suresh, P.; Thirumalaikolundusubramanian, P. Cardiovascular complications in ciguatera fish poisoning: A wake-up call. Heart Views Off. J. Gulf Heart Assoc. 2011, 12, 166. [CrossRef] [PubMed]

2. Albinali, H. Ciguatera fish poisoning. Heart Views 2011, 12, 165. [CrossRef] [PubMed] 
3. Accoroni, S.; Romagnoli, T.; Colombo, F.; Pennesi, C.; Di Camillo, C.G.; Marini, M.; Battocchi, C.; Ciminiello, P.; Dell'Aversano, C.; Dello Iacovo, E.; et al. Ostreopsis cf. ovata bloom in the northern Adriatic Sea during summer 2009: Ecology, molecular characterization and toxin profile. Mar. Pollut. Bull. 2011, 62, 2512-2519. [CrossRef] [PubMed]

4. Chinain, M.; Darius, H.; Ung, A.; Cruchet, P.; Wang, Z.; Ponton, D.; Laurent, D.; Pauillac, S. Growth and toxin production in the ciguatera-causing dinoflagellate Gambierdiscus polynesiensis (Dinophyceae) in culture. Toxicon 2010, 56, 739-750. [CrossRef]

5. Chinain, M.; Gatti, C.M.i.; Darius, H.T.; Quod, J.P.; Tester, P.A. Ciguatera poisonings: A global review of occurrences and trends. Harmful Algae 2021, 102, 101873. [CrossRef]

6. $\quad$ Friedman, M.; Fleming, L.; Fernandez, M.; Bienfang, P.; Schrank, K.; Dickey, R.; Bottein, M.; Backer, L.; Ayyar, R.; Weisman, R. Ciguatera fish poisoning: Treatment, prevention and management. Mar. Drugs 2008, 6, 456-479. [CrossRef]

7. Fleming, L.; Baden, D.; Bean, J.; Weisman, R.; Blythe, D. Marine Seafood Toxin Diseases: Issues in Epidemiology E Community Outreach; National Institute of Environmental Health Sciences: Durham, NC, USA, 1998.

8. WHO, F.A. Report of the Expert Meeting on Ciguatera poisoning: Rome, 19-23 November 2018; Food Safety and Quality Series; World Health Organization: Rome, Italy, 2020.

9. Parsons, M.; Richlen, M. An overview of ciguatera fish poisoning in the Bahamas. In The 15th Symposium on the Natural History of the Bahamas; Gerace Research Centre San Salvador Bahamas; A \& A Printing Inc.: Tampa, Florida, USA, 2016.

10. Chinain, M.; Gatti, C.; Roué, M.; Darius, H. Ciguatera-causing dinoflagellates in the genera gambierdiscus and fukuyoa: Distribution, ecophysiology and toxicology. In Dinoflagellates: Morphology, Life History and Ecological Significance; Subba Rao, D.V., Ed.; Nova Science Publishers, Inc.: Hauppauge, NY, USA, 2020; pp. 405-457.

11. Litaker, R.W.; Vandersea, M.W.; Faust, M.A.; Kibler, S.R.; Nau, A.W.; Holland, W.C.; Chinain, M.; Holmes, M.J.; Tester, P.A. Global distribution of ciguatera causing dinoflagellates in the genus Gambierdiscus. Toxicon 2010, 56, 711-730. [CrossRef]

12. Holmes, M. Gambierdiscus yasumotoi sp. nov.(Dinophyceae), a toxic benthic dinoflagellate from southeastern Asia. J. Phycol. 1998, 34, 661-668. [CrossRef]

13. Kashiwada, S.; Ishikawa, H.; Miyamoto, N.; Ohnishi, Y.; Magara, Y. Fish test for endocrine-disruption and estimation of water quality of Japanese rivers. Water Res. 2002, 36, 2161-2166. [CrossRef]

14. Nishimura, T.; Sato, S.; Tawong, W.; Sakanari, H.; Uehara, K.; Shah, M.; Suda, S.; Yasumoto, T.; Taira, Y.; Yamaguchi, H. Genetic diversity and distribution of the ciguatera-causing dinoflagellate Gambierdiscus spp.(Dinophyceae) in coastal areas of Japan. PLoS ONE 2013, 8, e60882. [CrossRef]

15. Rhodes, L.; Gimenez, P.; Smith, K.; Harwood, T. Gambierdiscus cf. yasumotoi (Dinophyceae) isolated from New Zealand's sub-tropical northern coastal waters. N. Z. J. Mar. Freshw. Res. 2014, 48, 303-310. [CrossRef]

16. Casabianca, S.; Perini, F.; Casabianca, A.; Battocchi, C.; Giussani, V.; Chiantore, M.; Penna, A. Monitoring toxic Ostreopsis cf. ovata in recreational waters using a qPCR based assay. Mar. Pollut. Bull. 2014, 88, 102-109. [CrossRef]

17. Cohu, S.; Thibaut, T.; Mangialajo, L.; Labat, J.P.; Passafiume, O.; Blanfune, A.; Simon, N.; Cottalorda, J.M.; Lemee, R. Occurrence of the toxic dinoflagellate Ostreopsis cf. ovata in relation with environmental factors in Monaco (NW Mediterranean). Mar. Pollut. Bull. 2011, 62, 2681-2691. [CrossRef]

18. Parsons, M.L.; Brandt, A.L.; Ellsworth, A.; Leynse, A.K.; Rains, L.K.; Anderson, D.M. Assessing the use of artificial substrates to monitor Gambierdiscus populations in the Florida Keys. Harmful Algae 2017, 68, 52-66. [CrossRef]

19. Yasumoto, T.; Nakajima, I.; Bagnis, R.; Adachi, R. Finding of a dinoflagellate as a likely culprit of ciguatera. Bull. Jpn. Soc. Sci. Fish. 1977, 43, 1021-1026. [CrossRef]

20. Yasumoto, T.; Inoue, A.; Bagnis, R. Ecological survey of a toxic dinoflagellate associated with ciguatera. In Toxic Dinoflagellate Blooms; Taylor, D.L., Seliger, H.H., Eds.; Elsevier North Holland, Inc.: New York, NY, USA, 1979; pp. $221-224$.

21. Yasumoto, T.; Inoue, A.; Ochi, T.; Fujimoto, K.; Oshima, Y.; Fukuyo, Y.; Adachi, R.; Bagnis, R. Environmental studies on a toxic dinoflagellate responsible for ciguatera. Bull. Jpn. Soc. Sci. Fish. 1980, 46, 1397-1404. [CrossRef]

22. Ballantine, D.L.; Tosteson, T.R.; Bardales, A.T. Population dynamics and toxicity of natural populations of benthic dinoflagellates in southwestern Puerto Rico. J. Exp. Mar. Biol. Ecol. 1988, 119, 201-212. [CrossRef]

23. Lobel, P.S.; Anderson, D.M.; Durand-Clement, M. Assessment of Ciguatera Dinoflagellate Populations: Sample Variability and Algal Substrate Selection. Biol. Bull. 1988, 175, 94-101. [CrossRef]

24. Bomber, J.W.; Rubio, M.G.; Norris, D.R. Epiphytism of dinoflagellates associated with the disease ciguatera: Substrate specificity and nutrition. Phycologia 1989, 28, 360-368. [CrossRef]

25. Litaker, R.; Vandersea, M.; Faust, M.; Kibler, S.; Chinain, M.; Holmes, M.; Holland, W.; Tester, P. Taxonomy of Gambierdiscus including four new species, Gambierdiscus caribaeus, Gambierdiscus carolinianus, Gambierdiscus carpenteri and Gambierdiscus ruetzleri (Gonyaulacales, Dinophyceae). Phycologia 2009, 48, 344-390. [CrossRef]

26. Rhodes, L.; Smith, K.; Murray, J.; Nishimura, T.; Finch, S. Ciguatera fish poisoning: The risk from an Aotearoa/New Zealand perspective. Toxins 2020, 12, 50. [CrossRef]

27. Biotoxins, F.M. FAO Food and Nutrition Paper 80; Food and Agriculture Organization of the United Nations: Rome, Italy, 2004.

28. De Rota, F. URDANETA $i$ CIENTÍFICO? Institute de Historia and Cultura Naval: Madrid, Spain, 2009; 38p.

29. Pearn, J. Around the rim: The role of surgeons in discovery and research in the Pacific rim. Part I. Surgeons in the Pacific: Expeditioners and expedition leaders. Aust. New Zealand J. Surg. 1994, 64, 38-44. [CrossRef]

30. Lee, C. Fish poisoning with particular reference to ciguatera. J. Trop. Med. Hyg. 1980, 83, 93-97. [PubMed] 
31. Silva, S. Contribution al'etude du microplankton de dakar et des regions maritimes voisines. Bull. Inst. Fr. Afr. Noire. Ser. A. Sci. Nat. 1956, 18, 335-371.

32. Guzman-Perez, S.; Park, D. Ciguatera toxins: Chemistry and detection. Food Sci. Technol. N.Y. Marcel Dekker 2000, 401-418.

33. Tester, P.A.; Litaker, R.W.; Berdalet, E. Climate change and harmful benthic microalgae. Harmful Algae 2020, 91, 101655. [CrossRef]

34. Landrigan, P.J.; Stegeman, J.J.; Fleming, L.E.; Allemand, D.; Anderson, D.M.; Backer, L.C.; Brucker-Davis, F.; Chevalier, N.; Corra, L.; Czerucka, D.; et al. Human Health and Ocean Pollution. Ann. Glob. Health 2020, 86, 1-64. [CrossRef]

35. Kibler, S.; Litaker, R.; Holland, W.; Vandersea, M.; Tester, P. Growth of eight Gambierdiscus (Dinophyceae) species: Effects of temperature, salinity and irradiance. Harmful Algae 2012, 19, 1-14. [CrossRef]

36. Gingold, D.B.; Strickland, M.J.; Hess, J.J. Ciguatera fish poisoning and climate change: Analysis of National Poison Center Data in the United States, 2001-2011. Environ. Health Perspect. 2014, 122, 580-586. [CrossRef] [PubMed]

37. Kibler, S.R.; Tester, P.A.; Kunkel, K.E.; Moore, S.K.; Litaker, R.W. Effects of ocean warming on growth and distribution of dinoflagellates associated with ciguatera fish poisoning in the Caribbean. Ecol. Model. 2015, 316, 194-210. [CrossRef]

38. Yong, H.L.; Mustapa, N.I.; Lee, L.K.; Lim, Z.F.; Tan, T.H.; Usup, G.; Gu, H.; Litaker, R.W.; Tester, P.A.; Lim, P.T. Habitat complexity affects benthic harmful dinoflagellate assemblages in the fringing reef of Rawa Island, Malaysia. Harmful Algae 2018, 78, 56-68. [CrossRef]

39. Hallegraeff, G.M.; Anderson, D.M.; Belin, C.; Bottein, M.-Y.D.; Bresnan, E.; Chinain, M.; Enevoldsen, H.; Iwataki, M.; Karlson, B.; McKenzie, C.H.; et al. Perceived global increase in algal blooms is attributable to intensified monitoring and emerging bloom impacts. Commun. Earth Environ. 2021, 2, 117. [CrossRef]

40. Rhodes, L.; Smith, K.; Munday, R.; Selwood, A.; McNabb, P.; Holland, P.; Bottein, M. Toxic dinoflagellates (Dinophyceae) from Rarotonga, Cook Islands. Toxicon 2010, 56, 751-758. [CrossRef]

41. Uddin, S.; Sultan, M.; Behbehani, M.; Al-Rashed, W.; Al-Shamroukh, D.; Al-Khabbaz, A.; Al-Yaegoub, A.; Al-Bahloul, M. A Remote Sensing-Based Early Warning System for Algal Blooms in Kuwait Bay and Coastal Waters; Project EM033C, Final Report, KISR12320; Kuwait Institute for Scientific Research, 2014; 145p.

42. Saburova, M.; Polikarpov, I.; Al-Yamani, F. New records of the genus Gambierdiscus in marginal seas of the Indian Ocean. Mar. Biodivers. Rec. 2013, 6, e91. [CrossRef]

43. Catania, D. The Prevalence of Benthic Dinoflagellates Associated with Ciguatera in the Central Red Sea. M.Sc. Thesis, King Abdullah University of Science and Technology, Thuwal, Saudi Arabia, 2012; 40p.

44. Catania, D.; Richlen, M.; Mak, Y.; Morton, S.; Laban, E.; Xu, Y.; Anderson, D.; Chan, L.; Berumen, M. The prevalence of benthic dinoflagellates associated with ciguatera fish poisoning in the central Red Sea. Harmful Algae 2017, 68, 206-216. [CrossRef] [PubMed]

45. Manche, C. A Remote Sensing Based Early Warning System for Algal Blooms in Kuwait Bay and Coastal Waters. Master's Thesis, Western Michigan University, Kalamazoo, MI, USA, 2014. Available online: https://scholarworks.wmich.edu/masters_theses/ 546 (accessed on 1 June 2021).

46. Quod, J.-P.; Turquet, J.; Conejero, S.; Ralijaona, C. Ciguatera risk assessment in the Indian Ocean following the 1998 coral bleaching event. Coral Reef Degrad. Indian Ocean. Status Rep. 2000, 166-168.

47. Bagnis, R. L'ichtyosarcotoxisme de type ciguatera: Phénomène complexe de biologie marine et humaine. Oceanol. Acta 1981, 4 , 375-387.

48. Behbehani, M.; Uddin, S.; Dupont, S.; Sajid, S.; Al-Musalam, L.; Al-Ghadban, A. Response of corals Acropora pharaonis and Porites lutea to changes in $\mathrm{pH}$ and temperature in the Gulf. Sustainability 2019, 11, 3156. [CrossRef]

49. Reguera, B.; Alonso, R.; Moreira, A.; Méndez, S.; Dechraoui-Bottein, M.-Y. Guide for Designing and Implementing a Plan to Monitor Toxin-Producing Microalgae. In Intergovermental Oceanographic Commission Manuals and Guides, 2nd ed.; UNESCO: Paris, France; IAEA: Vienna, Austria, 2016; 66p, Available online: http:/ / hdl.handle.net/11329/304 (accessed on 1 June 2021).

50. Jean Turquet, J.-P.Q.; Ten-Hage, L.; Dahalani, Y.; Wendling, B. Example of a Gambierdiscus toxicus flare-up following the 1998 coral bleaching event in Mayotte Island (Comoros, south-west Indian Ocean). In Proceedings of the Harmful Algal Blooms 2000: Proceedings of the Ninth International Conference on Harmful Algal Blooms, Hobart, Australia, 7-11 February 2000.

51. Uddin, S.; Gevao, B.; Al-Ghadban, A.; Nithyanandan, M.; Al-Shamroukh, D. Acidification in Arabian Gulf-Insights from pH and temperature measurements. J. Environ. Monit. 2012, 14, 1479-1482. [CrossRef]

52. Al-Musalam, L.; Uddin, S. Effect of Ocean Acidification on Growth and Abundance of Penaeus Semisulcatus in the Northern Arabian Gulf; Annual Progress Report No. 1(EM078C); Kuwait Insitute for Scientific Research: Kuwait City, Kuwait, 2018; 27p.

53. Al-Musallam, L.; Uddin, S.; Al-Dakkor, S.; Kumar, V. Effect of ocean acidification and ocean warming on the growth and survival of Penaeus semisulcatus Post-Larvae. J Earth Env. Sci 2019, 3. [CrossRef]

54. Elkadiri, R.; Manche, C.; Sultan, M.; Al-Dousari, A.; Uddin, S.; Chouinard, K.; Abotalib, A.Z. Development of a Coupled Spatiotemporal Algal Bloom Model for Coastal Areas: A Remote Sensing and Data Mining-Based Approach. IEEE J. Sel. Top. Appl. Earth Obs. Remote Sens. 2016, 9, 5159-5171. [CrossRef]

55. Heil, C.A.; Glibert, P.M.; Al-Sarawi, M.A.; Faraj, M.; Behbehani, M.; Husain, M. First record of a fish-killing Gymnodinium sp. bloom in Kuwait Bay, Arabian Sea: Chronology and potential causes. Mar. Ecol. Prog. Ser. 2001, 214, 15-23. [CrossRef]

56. Glibert, P.; Landsberg, J.; Evans, J.; Al-Sarawi, M.; Faraj, M.; Al-Jarallah, M.; Haywood, A.; Ibrahem, S.; Klesius, P.; Powell, C. A fish kill of massive proportion in Kuwait Bay, Arabian Gulf, 2001: The roles of bacterial disease, harmful algae, and eutrophication. Harmful Algae 2002, 1, 215-231. [CrossRef] 
57. Walsh, J.; Steidinger, K. Saharan dust and Florida red tides: The cyanophyte connection. J. Geophys. Res. Ocean. 2001, 106, 11597-11612. [CrossRef]

58. Richlen, M.L.; Morton, S.L.; Jamali, E.A.; Rajan, A.; Anderson, D.M. The catastrophic 2008-2009 red tide in the Arabian gulf region, with observations on the identification and phylogeny of the fish-killing dinoflagellate Cochlodinium polykrikoides. Harmful Algae 2010, 9, 163-172. [CrossRef]

59. Berland, B.; Grzebyk, D.; Thomassin, B.-A. Benthic dinoflagellates from the coral reef lagoon of Mayotte Island (SW Indian Ocean); identification, toxicity and preliminary ecophysiological study. Bull.-Soc. Pathol. Exot. 1992, 85, 453. [PubMed]

60. Grzebyk, D.; Berland, B.; Thomassin, B.A.; Bosi, C.; Arnoux, A. Ecology of ciguateric dinoflagellates in the coral reef complex of Mayotte Island (SW Indian Ocean). J. Exp. Mar. Biol. Ecol. 1994, 178, 51-66. [CrossRef]

61. Thomassin, B.; ME, A.H.; Quod, J.; Maggiorani, J.; Berland, B.; Grzebyk, D.; Coqueugniot, J. Evolution of Gambierdiscus toxicus populations in the coral reef complex of Mayotte Island (SW Indian Ocean) during the 1985-1991 period. Bull. Soc. Pathol. Exot. 1992, 85, 449-452.

62. Quod, J.; Prunaux, O.; Guignard, A. Les empoisonnements par poissons tropicaux à La Réunion: Synthèse et perspectives. Rev. Méd. Vét. 1990, 141, 1005-1009.

63. Quod, J.; Bourdeau, P.; Turquet, J.; Guignard, A. La ciguatera dans les DOM-TOM: Aspects épidémiologiques et physiopathologiques. Rev. Méd. Vét. 1994, 170, 141-146.

64. Quod, J.; Turquet, J. Ciguatera in Reunion Island (SW Indian Ocean): Epidemiology and clinical patterns. Toxicon 1996, 34, 779-785. [CrossRef]

65. Ten-Hage, L.; Turquet, J.; Quod, J.-P.; Couté, A. An overview of the biodiversity of benthic diniflagellates from La Reunion Island (France, South-west Indian Ocean). In 9th Int Conference on Harmful Algal Blooms (HAB 2000); Hobart, Tasmania, 2000.

66. Lugomela, C. Autecology of the Toxic Dinoflagellate Gambierdiscus toxicus Adachi et Fukyo (Dinophyceae) in Central Coastal Areas of Tanzania. West. Indian Ocean J. Mar. Sci. 2006, 5, 213-221. [CrossRef]

67. Gómez, F.; Qiu, D.; Lopes, R.M.; Lin, S. Fukuyoa paulensis gen. et sp. nov., a New Genus for the Globular Species of the Dinoflagellate Gambierdiscus (Dinophyceae). PLoS ONE 2015, 10, e0119676. [CrossRef]

68. Saburova, M.; Igor, P.; Al-Yamani, F. Gambierdiscus in Kuwait. Harmful Algae News 2013, 47, $22-23$.

69. Munir, S.; Siddiqui, P.; Morton, S.L. The occurrence of the ciguatera fish poisoning producing dinoflagellate genus Gambierdiscus in Pakistan waters. Algae 2011, 26, 317-325. [CrossRef]

70. Polikarpov, I.; Saburova, M.; Al-Yamani, F. Diversity and distribution of winter phytoplankton in the Arabian Gulf and the Sea of Oman. Cont. Shelf Res. 2016, 119, 85-99. [CrossRef]

71. Naik, R.K.; Hegde, S.; Anil, A.C. Dinoflagellate community structure from the stratified environment of the Bay of Bengal, with special emphasis on harmful algal bloom species. Environ. Monit. Assess. 2011, 182, 15-30. [CrossRef]

72. Rajeish, M.; Shekar, M.; Madhushree, H.; Venugopal, M. Presumptive case of ciguatera fish poisoning in Mangalore, India. Curr. Sci. 2016, 111, 1543-1547. [CrossRef]

73. Rajisha, R.; Kishore, P.; Panda, S.K.; Ravishankar, C.N.; Kumar, K.A. Confirmation of ciguatoxin fish poisoning in red snapper, Lutjanus bohar (Forsskål, 1775) by mouse bioassay. Fish. Technol. 2017, 54, 287-290.

74. Karunasagar, I.; Turner, A.D.; Maskrey, B.; Robertson, A.; Shivanagouda Hosagoudar, S.; Rai, P.; Adappa, S.; Hiremath, S.; Kogaluru Shivakumaraswamy, S.; Dechraoui Bottein, M.Y.; et al. Report of a major outbreak of ciguatera fish poisoning in Mangalore, India. In Proceedings of the 18th International Conference on Harmful Algae; Rome, Italy, $2018 ;$ p. 482.

75. Rajisha, R.; Kishore, P.; Panda, S.K.; Harikrisnan, G.; Ajitha, K.C.; Suresh, M.K.; Chowdhury, L.M.; Ravishankar, C.N.; Kumar, K.A. Incidence of ciguatoxin fish poisoning in Trivandrum, India. Indian J. Fish. 2017, 64, 129-133. [CrossRef]

76. Hamilton, B.; Hurbungs, M.; Jones, A.; Lewis, R.J. Multiple ciguatoxins present in Indian Ocean reef fish. Toxicon 2002, 40, 1347-1353. [CrossRef]

77. Hamilton, B.; Hurbungs, M.; Vernoux, J.-P.; Jones, A.; Lewis, R.J. Isolation and characterisation of Indian Ocean ciguatoxin. Toxicon 2002, 40, 685-693. [CrossRef]

78. Diogène, J.; Reverté, L.; Rambla-Alegre, M.; Del Río, V.; De La Iglesia, P.; Campàs, M.; Palacios, O.; Flores, C.; Caixach, J.; Ralijaona, C. Identification of ciguatoxins in a shark involved in a fatal food poisoning in the Indian Ocean. Sci. Rep. 2017, 7, 1-8. [CrossRef]

79. Roeder, K.; Erler, K.; Kibler, S.; Tester, P.; Van The, H.; Nguyen-Ngoc, L.; Gerdts, G.; Luckas, B. Characteristic profiles of Ciguatera toxins in different strains of Gambierdiscus spp. Toxicon 2010, 56, 731-738. [CrossRef] [PubMed]

80. Pisapia, F.; Holland, W.C.; Hardison, D.R.; Litaker, R.W.; Fraga, S.; Nishimura, T.; Adachi, M.; Nguyen-Ngoc, L.; Séchet, V.; Amzil, Z.; et al. Toxicity screening of 13 Gambierdiscus strains using neuro-2a and erythrocyte lysis bioassays. Harmful Algae 2017, 63, 173-183. [CrossRef]

81. Friedemann, M. Ciguatera fish poisoning outbreaks from 2012 to 2017 in Germany caused by snappers from India, Indonesia, and Vietnam. J. Consum. Prot. Food Saf. 2019, 14, 71-80. [CrossRef]

82. Yogi, K.; Oshiro, N.; Inafuku, Y.; Hirama, M.; Yasumoto, T. Detailed LC-MS/MS Analysis of Ciguatoxins Revealing Distinct Regional and Species Characteristics in Fish and Causative Alga from the Pacific. Anal. Chem. 2011, 83, 8886-8891. [CrossRef] [PubMed] 
83. Clausing, R.; Chinain, M.; Bottein, M. Practical Sampling Guidance for Determination of Ciguatoxin in Fish. In Guide for Designing and Implementing a Plant to Monitor Toxin-Producing Microalgae, 2nd ed.; IOC Manuals and Guides, no. 59; Intergovernmental Oceanographic Commission (IOC) of UNESCO: Paris, France; International Atomic Energy Agency (IAEA): Vienna, Austria, 2016; pp. 51-63.

84. Hurbungs, M.D.; Jayabalanm, N.; Chineah, V. Seasonal distribution of potentially toxic benthic dinoflagellates in the lagoon of Trou aux Biches, Mauritius. In Proceedings of the 5th Annual Meeting of Agricultural Scientists, Reduit, Mauritius, 3-4 May 2001; Lalouette, J.A., Bachraz, D.Y., Eds.; The Food and Agricultural Research Council: Reduit, Mauritius, 2002; pp. $211-217$.

85. Quod, J.; Turquet, J.; Diogene, G.; Fessard, V. Screening of extracts of dinoflagellates from coral reefs (Reunion Island, SW Indian Ocean), and their biological activities. Harmful Mar. Algal Bloom. 1995, 815-820.

86. Turquet, J. Assemblage of benthic dinofagellates and monitoring of harmful species in Reunion Island, SW Indian Ocean, 1993-1996. Harmful Algae 1998, 44-47.

87. Roué, M.; Smith, K.; Sibat, M.; Viallon, J.; Henry, K.; Ung, A.; Biessy, L.; Hess, P.; Darius, H.; Chinain, M. Assessment of Ciguatera and Other Phycotoxin-Related Risks in Anaho Bay (Nuku Hiva Island, French Polynesia): Molecular, Toxicological, and Chemical Analyses of Passive Samplers. Toxins 2020, 12, 321. [CrossRef] [PubMed]

88. Smith, K.; Kohli, G.; Murray, S.; Rhodes, L. Assessment of the metabarcoding approach for community analysis of benthicepiphytic dinoflagellates using mock communities. N. Z. J. Mar. Freshw. Res. 2017, 51, 555-576. [CrossRef]

89. Rhodes, L.; Smith, K.; Verma, A.; Murray, S.; Harwood, D.; Trnski, T. The dinoflagellate genera Gambierdiscus and Ostreopsis from subtropical Raoul Island and North Meyer Island, Kermadec Islands. N. Z. J. Mar. Freshw. Res. 2017, 51, 490-504. [CrossRef]

90. Hoppenrath, M.; Kretzschmar, A.; Kaufmann, M.; Murray, S. Morphological and molecular phylogenetic identification and record verification of Gambierdiscus excentricus (Dinophyceae) from Madeira Island (NE Atlantic Ocean). Mar. Biodivers. Rec. 2019, 12, 1-9. [CrossRef]

91. Rhodes, L.; Smith, K. A checklist of the benthic and epiphytic marine dinoflagellates of New Zealand, including Rangitāhua/Kermadec Islands. N. Z. J. Mar. Freshw. Res. 2019, 53, 258-277. [CrossRef]

92. Faust, M. Observation of sand-dwelling toxic dinoflagellates (Dinophyceae) from widely differing sites, including two new species. J. Phycol. 1995, 31, 996-1003. [CrossRef]

93. Adachi, R.; Fukuyo, Y. The thecal structure of a marine toxic dinoflagellate Gambierdiscus toxicus gen. et sp. nov. collected in a ciguatera endemic area. Bull. Jpn. Soc. Sci. Fish. 1979, 45, 67-71. [CrossRef]

94. Chinain, M.; Faust, M.A.; Pauillac, S. Morphology and molecular analyses of three toxic species of Gambierdiscus (Dinophyceae): G. pacificus sp. nov., G. australes, sp. nov. and G. polynesiensis, sp. nov. J. Phycol. 1999, 35, 1282-1296. [CrossRef]

95. Xu, Y.; Richlen, M.L.; Morton, S.L.; Mak, Y.L.; Chan, L.L.; Tekiau, A.; Anderson, D.M. Distribution, abundance and diversity of Gambierdiscus spp. from a ciguatera-endemic area in Marakei, Republic of Kiribati. Harmful Algae 2014, 34, 56-68. [CrossRef]

96. Dai, X.; Mak, Y.; Lu, C.; Mei, H.; Wu, J.; Lee, W.; Chan, L.; Lim, P.; Mustapa, N.; Lim, H. Taxonomic assignment of the benthic toxigenic dinoflagellate Gambierdiscus sp. type 6 as Gambierdiscus balechii (Dinophyceae), including its distribution and ciguatoxicity. Harmful Algae 2017, 67, 107-118. [CrossRef]

97. Richlen, M.; Morton, S.; Barber, P.; Lobel, P. Phylogeography, morphological variation and taxonomy of the toxic dinoflagellate Gambierdiscus toxicus (Dinophyceae). Harmful Algae 2008, 7, 614-629. [CrossRef]

98. Fraga, S.; Rodríguez, F.; Caillaud, A.; Diogène, J.; Raho, N.; Zapata, M. Gambierdiscus excentricus sp. nov.(Dinophyceae), a benthic toxic dinoflagellate from the Canary Islands (NE Atlantic Ocean). Harmful Algae 2011, 11, 10-22. [CrossRef]

99. Fraga, S.; Rodríguez, F. Genus Gambierdiscus in the Canary Islands (NE Atlantic Ocean) with description of Gambierdiscus silvae sp. nov., a new potentially toxic epiphytic benthic dinoflagellate. Protist 2014, 165, 839-853. [CrossRef] [PubMed]

100. Fraga, S.; Rodríguez, F.; Riobó, P.; Bravo, I. Gambierdiscus balechii sp. nov (Dinophyceae), a new benthic toxic dinoflagellate from the Celebes Sea (SW Pacific Ocean). Harmful Algae 2016, 58, 93-105. [CrossRef] [PubMed]

101. Nascimento, S.; Melo, G.; Salgueiro, F.; Diniz, B.; Fraga, S. Morphology of Gambierdiscus excentricus (Dinophyceae) with emphasis on sulcal plates. Phycologia 2015, 54, 628-639. [CrossRef]

102. Rodríguez, F.; Fraga, S.; Ramilo, I.; Rial, P.; Figueroa, R.; Riobó, P.; Bravo, I. Canary Islands (NE Atlantic) as a biodiversity 'hotspot' of Gambierdiscus: Implications for future trends of ciguatera in the area. Harmful Algae 2017, 67, 131-143. [CrossRef] [PubMed]

103. Leung, P.; Yan, M.; Lam, V.; Yiu, S.; Chen, C.; Murray, J.; Harwood, D.; Rhodes, L.; Lam, P.; Wai, T. Phylogeny, morphology and toxicity of benthic dinoflagellates of the genus Fukuyoa (Goniodomataceae, Dinophyceae) from a subtropical reef ecosystem in the South China Sea. Harmful Algae 2018, 74, 78-97. [CrossRef]

104. Rhodes, L.; Smith, K.; Verma, A.; Curley, B.; Harwood, D.; Murray, S.; Kohli, G.; Solomona, D.; Rongo, T.; Munday, R. A new species of Gambierdiscus (Dinophyceae) from the south-west Pacific: Gambierdiscus honu sp. nov. Harmful Algae 2017, 65, 61-70. [CrossRef] [PubMed]

105. Smith, K.; Biessy, L.; Argyle, P.; Trnski, T.; Halafihi, T.; Rhodes, L. Molecular identification of Gambierdiscus and Fukuyoa (Dinophyceae) from environmental samples. Mar. Drugs 2017, 15, 243. [CrossRef]

106. Tudó, À.; Toldrà, A.; Rey, M.; Todolí, I.; Andree, K.; Fernández-Tejedor, M.; Campàs, M.; Sureda, F.; Diogène, J. Gambierdiscus and Fukuyoa as potential indicators of ciguatera risk in the Balearic Islands. Harmful Algae 2020, 99, 101913. [CrossRef]

107. Bravo, I.; Rodríguez, F.; Ramilo, I.; Afonso-Carrillo, J. Epibenthic Harmful Marine Dinoflagellates from Fuerteventura (Canary Islands), with Special Reference to the Ciguatoxin-Producing Gambierdiscus. J. Mar. Sci. Eng. 2020, 8, 909. [CrossRef] 
108. Jang, S.H.; Jeong, H.J.; Yoo, Y.D. Gambierdiscus jejuensis sp. nov., an epiphytic dinoflagellate from the waters of Jeju Island, Korea, effect of temperature on the growth, and its global distribution. Harmful Algae 2018, 80, 149-157. [CrossRef]

109. Nishimura, T.; Sato, S.; Tawong, W.; Sakanari, H.; Yamaguchi, H.; Adachi, M. Morphology of G ambierdiscus scabrosus sp. nov.(G onyaulacales): A new epiphytic toxic dinoflagellate from coastal areas of Japan. J. Phycol. 2014, 50, 506-514. [CrossRef] [PubMed]

110. Tawong, W.; Nishimura, T.; Sakanari, H.; Sato, S.; Yamaguchi, H.; Adachi, M. Characterization of G ambierdiscus and C oolia (D inophyceae) isolates from T hailand based on morphology and phylogeny. Phycol. Res. 2015, 63, 125-133. [CrossRef]

111. Larsson, M.; Harwood, T.; Lewis, R.; SWA, H.; Doblin, M. Toxicological characterization of Fukuyoa paulensis (Dinophyceae) from temperate Australia. Phycol. Res. 2019, 67, 65-71. [CrossRef]

112. Zhang, H.; Wu, Z.; Cen, J.; Li, Y.; Wang, H.; Lu, S. First report of three benthic dinoflagellates, Gambierdiscus pacificus, G. australes and G. caribaeus (Dinophyceae), from Hainan Island, South China Sea. Phycol. Res. 2016, 64, 259-273. [CrossRef]

113. Kretzschmar, A.; Verma, A.; Harwood, T.; Hoppenrath, M.; Murray, S. Characterization of Gambierdiscus lapillus sp. nov.(Gonyaulacales, Dinophyceae): A new toxic dinoflagellate from the Great Barrier Reef (Australia). J. Phycol. 2017, 53, 283-297. [CrossRef] [PubMed]

114. Murray, S.; Momigliano, P.; Heimann, K.; Blair, D. Molecular phylogenetics and morphology of Gambierdiscus yasumotoi from tropical eastern Australia. Harmful Algae 2014, 39, 242-252. [CrossRef]

115. Dickey, R.; Plakas, S. Ciguatera: A public health perspective. Toxicon 2010, 56, 123-136. [CrossRef]

116. Wasay, M.; Sarangzai, A.; Siddiqi, A.; Nizami, Q. Ciguatera fish poisoning with elevated muscle enzymes and abnormal spinal MRI. Southeast Asian J. Trop. Med. Public Health 2008, 39, 307.

117. Rajisha, R.; Kishore, P.; Panda, S.; Kumar, K.A. Ciguatoxin-An Emerging Biological Hazard among Reef Fishes of India. Fish. Tech. 2018, 55, 153-167.

118. Saraya, A.; Sintunawa, C.; Wacharapluesadee, S.; Swangpun, K.; Dumrongchua, S.; Wilde, H.; Hemachudha, T. Marine Fish Toxins in Thailand: Report of 6 Suspected Ciguatera Cases. Case Rep. Clin. Med. 2014, 3, 7. [CrossRef]

119. Sozzi, G.; Marotta, P.; Aldeghi, D.; Tredici, G.; Calvi, L. Polyneuropathy Secondary to Ciguatoxin Poisoning. Ital. J. Neurol. Sci. 1988, 9, 491-495. [CrossRef] [PubMed]

120. De Haro, L.; Pommier, P.; Valli, M. Emergence of Imported Ciguatera in Europe: Report of 18 Cases at the Poison Control Centre of Marseille. J. Toxicol. Clin. Toxicol. 2003, 41, 927-930. [CrossRef]

121. Habermehl, G.G.; Krebs, H.C.; Rasoanaivo, P.; Ramialiharisoa, A. Severe ciguatera poisoning in Madagascar: A case report. Toxicon 1994, 32, 1539-1542. [CrossRef]

122. Anonymous. Mangaluru: Khader Warns against Consumption of Red Snapper, Puffer Fish Heads, in Daijizorld Published on 6 October 2016: Mangaluru, India. Available online: https: / / www.daijiworld.com/news/newsDisplay?newsID=417564 (accessed on 1 June 2021).

123. Jin, D.; Kite-Powell, H.; Hoagland, P.; Solow, A. A bioeconomic analysis of traditional fisheries in the Red Sea. Mar. Resour. Econ. 2012, 27, 137-148. [CrossRef]

124. Anonymous. Fishbase. Available online: https:/ /www.fishbase.de (accessed on 1 June 2021).

125. Mattei, C.; Vetter, I.; Eisenblätter, A.; Krock, B.; Ebbecke, M.; Desel, H.; Zimmermann, K. Ciguatera fish poisoning: A first epidemic in Germany highlights an increasing risk for European countries. Toxicon 2014, 91, 76-83. [CrossRef] [PubMed] 